ARGONNE NATIONAL LABORATORY

9700 South Cass Avenue

Argonne, IL 60439

ANL/MCS-TM-233

\title{
Using ADIFOR and ADIC to Provide Jacobians for the SNES Component of PETSc
}

\author{
by \\ Po-Ting Wu, ${ }^{*}$ Christian H. Bischof, and Paul D. Hovland \\ e-mail: \{psu,bischof,hovland\}@mcs.anl.gov
}

Mathematics and Computer Science Division

Technical Memorandum No. 233

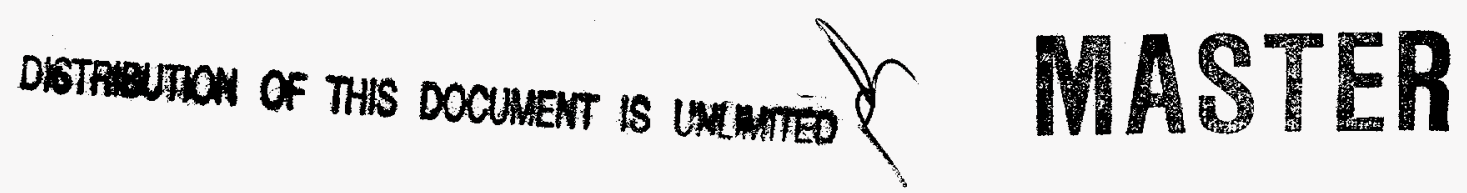

November 1997

This work was supported by the Mathematical, Information, and Computational Sciences Division subprogram of the Office of Computational and Technology Research, U.S. Department of Energy, under Contract W-31-109-Eng-38.

\footnotetext{
*Current address: M/S MPK16-102, Sun Microsystems, 2550 Garcia Avenue, Mountain View, CA 94043, (415) 786-9507, e-mail: po-ting.wu@Eng.Sun.COM.
} 


\section{DISCLAIMER}

This report was prepared as an account of work sponsored by an agency of the United States Government. Neither the United States Government nor any agency thereof, nor any of their employees, make any warranty, express or implied, or assumes any legal liabili. ty or responsibility for the accuracy, completeness, or usefulness of any information, apparatus, product, or process disclosed, or represents that its use would not infringe privately owned rights. Reference herein to any specific commercial product, process, or service by trade name, trademark, mamufacturer, or otherwise does not necessarily constitute or imply its endorsement, recommendation, or favoring by the United States Government or any agency thereof. The views and opinions of authors expressed herein do not necessarily state or reflect those of the United States Government or any agency thereof. 


\section{DISCLAIMER}

Portions of this document may be illegible electronic image products. Images are produced from the best available original document. 


\section{Contents}

$\begin{array}{ll}\text { Abstract } & 1\end{array}$

1 Introduction $\quad 1$

2 Outline of Procedure $\quad 1$

3 Isolating ADFunction $\quad 3$

4 Running ADIFOR/ADIC $\quad 3$

5 Constructing FormJacobian $\quad 7$

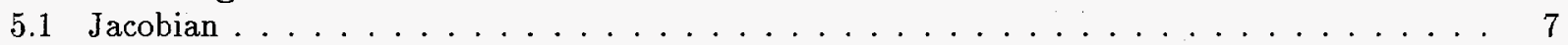

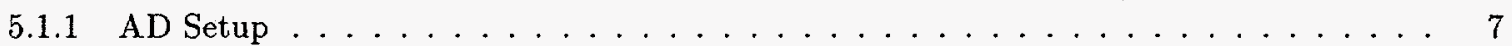

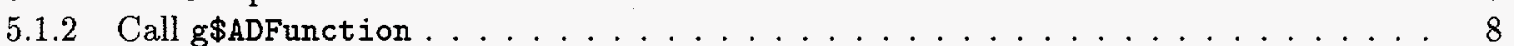

5.1.3 Transfer AD-generated Jacobian into PETSc Data Structures . . . . . . . . . . . . . 8

5.2 Sparse Jacobian . . . . . . . . . . . . . . . . . . . . . . . . 9

5.2 .1 AD Setup . . . . . . . . . . . . . . . . . . . . 9

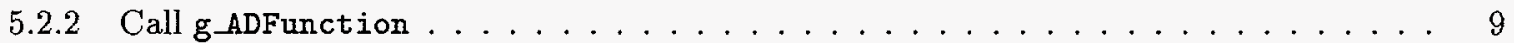

5.2 .3 Transfer AD-generated Jacobian into PETSc Data Structures . . . . . . . . . . . . 9

5.3 Compressed Jacobian . . . . . . . . . . . . . . . . . . . . . . . . . . 10

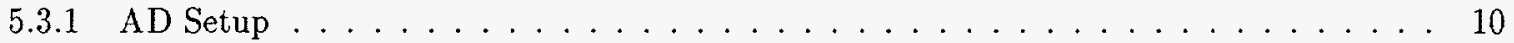

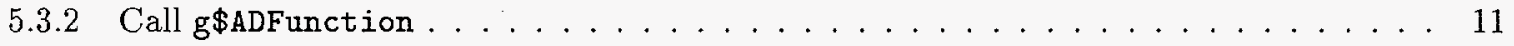

5.3 .3 Determine Whether Sparsity Pattern Has Changed . . . . . . . . . . . . . . . 11

5.3.4 Transfer AD-generated Jacobian into PETSc Data Structures . . . . . . . . . . . . . 11

5.4 ADIC Jacobian . . . . . . . . . . . . . . . . . . . . . . . . . . . . 12

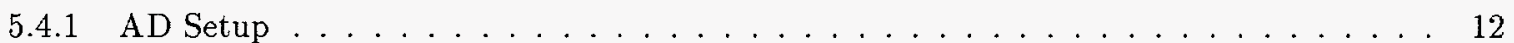

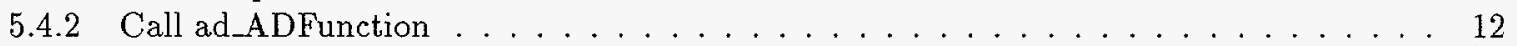

5.4 .3 Transfer AD-generated Jacobian into PETSc Data Structures . . . . . . . . . . . . 12

6 Summary $\quad 13$

$\begin{array}{ll}\text { Appendix } & 14\end{array}$

$\begin{array}{ll}\text { A g\$ADFunction } & 14\end{array}$

$\begin{array}{ll}B \text { g_ADFunction } & 16\end{array}$

$\begin{array}{lr}C \text { ad_ADFunction } & 18\end{array}$

$\begin{array}{lr}\text { D Dense FormJacobian } & 20\end{array}$

E Sparse FormJacobian $\quad 23$

$\begin{array}{lr}\text { F Colored FormJacobian } & 26\end{array}$

G ADIC FormJacobian $\quad 36$

$\begin{array}{ll}\text { References } & 39\end{array}$ 
Using ADIFOR and ADIC to Provide Jacobians for the SNES Component of PETSc

by

Po-Ting Wu, Christian H. Bischof, and Paul D. Hovland

\begin{abstract}
The solution of large-scale nonlinear problems is important to many areas of computational science. The SNES component of PETSc provides a robust and flexible suite of numerical routines for the solving such problems. These routines generally utilize the Jacobian matrix. We present a strategy for using ADIFOR or ADIC to assist in the development of a subroutine for computing this matrix. We illustrate this strategy using one of the PETSc example programs and four different approaches to computing the Jacobian via automatic differentiation.
\end{abstract}

\title{
1 Introduction
}

The solution of large-scale nonlinear problems pervades many facets of computational science and demands robust and flexible solution strategies. The SNES component of PETSc [2] provides a powerful suite of datastructure-neutral numerical routines for such problems. These routines generally require some derivative matrix, such as the Jacobian or, when the nonlinear system arises from optimization, a Hessian. These derivatives can be approximated with finite differences. However, the program will typically be more efficient and accurate if the programmer supplies a subroutine to compute the derivatives. Unfortunately, such a subroutine is often very complicated and difficult to program correctly by hand. We describe how automatic differentiation (AD) tools such as ADIFOR [3] and ADIC [6] can be utilized within the PETSc framework to automatically generate the subroutine for computing the derivatives. To describe this procedure, we use a simple problem from the PETSc SNES examples.

\section{Outline of Procedure}

We refer to the user-defined routines utilized by PETSc nonlinear solvers as FormFunction and FormJacobian, which compute the nonlinear function $F(X)$ and the Jacobian matrix $d F(X, d X)$, respectively. The routine FormFunction often includes several calls to PETSc routines before and after the actual function computation. These calls take care of data structure setup and communication, enabling a completely local function computation. It is this local function that we will differentiate using $A D$, and for that reason we will refer to it as ADFunction. After differentiating this routine using AD to form g_ADFunction, we build the FormJacobian routine. This procedure is illustrated in Figure 1 and outlined below.

1. Within the FormFunction routine, separate the PETSc pre- and postdeclaration from the local function evaluation. Extract the function computation into routine ADFunction.

2. Construct a composition file that lists the names of all of the source files that constitute the function evaluation program.

3. Construct a script file that tells ADIFOR/ADIC to differentiate the procedure named ADFunction with the independent variable $X$ and the dependent variable $F$, namely, to generate code to compute the derivative $\frac{d F}{d X}$, where $F$ is computed from $X$ by procedure ADFunction.

4. Execute the $\mathrm{AD}$ tools command, for example,

or

Adifor2.0 AD_SCRIPT=ad_petsc.adf

adic $-x v-d$ gradient $-i$ ad_petsc.init $-u$ 


\section{FormFunction(snes, X, F, user) \\ PETSc Pre-Declaration \\ ... \\ call ADF unction (X, F, corner, user) \\ ... \\ PETSc Post-Declaration}

$\operatorname{ADFunction}(\mathrm{X}, \mathrm{F}$, corner, user)

...

Evaluate $\mathrm{F}(\mathrm{X})$

...

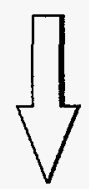

ADIFOR Script File:

$$
\begin{gathered}
\text { AD_TOP }=\text { ADFunction } \\
\text { AD_IVARS }=\mathrm{X} \\
\text { AD_OVARS }=\mathrm{F} \\
\ldots
\end{gathered}
$$
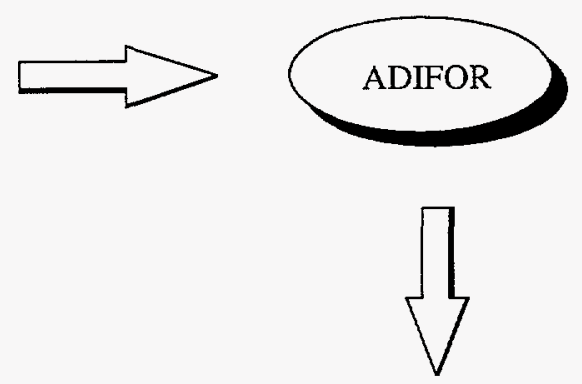

Form Jacobian(snes, X, jac, B, flag, user)

PETSc Pre-Declaration

ADIFOR Declaration

...

call $g_{-} A D F u n c t i o n(X, d X, F, d F$, corner, user)

Store Derivatives into PETSc matrix

...

PETSc Post-Declaration

Figure 1: Integration of PETSc and ADIFOR 
where ad_petsc.adf (for ADIFOR) and ad_petsc.init (for ADIC) are the names of the script files defined in the previous step. This process creates a file that contains the derivative routine g_ADFunction (for ADIFOR) or ad_ADFunction (for ADIC).

5. Create the FormJacobian routine:

(a) Incorporate the PETSc predeclaration.

(b) Set up the derivative computation.

(c) Invoke g_ADFunction (for ADIFOR) or ad_ADFunction (for ADIC) with a user-specified value of $X$ to compute the value of $F$ and $\frac{d F}{d X}$.

(d) Copy derivatives into PETSc data structures.

(e) Incorporate the PETSc postdeclaration.

\section{Isolating ADFunction}

Consider the version of FormFunction in Figure 2. This is the Fortran version of SNES example 5 that is part of the standard PETSc distribution (\$PETSC_DIR/src/snes/examples/tutorials/ex5f.F). We have inserted comments to denote the beginning and end of the local function computation. This code can be extracted into the routine ADFunction, as shown in Figure 3. The calls to PETSc routines surrounding this code make up the PETSC pre- and postdeclarations, as shown in Figures 4 and 5 . A similar procedure can be employed for the $\mathrm{C}$ version. We note that newer versions of PETSc include a version of this example where most of the local function computation is encapsulated in the routine Applicationfunction. However, the older version presented here seemed more appropriate for didactic purposes.

\section{Running ADIFOR/ADIC}

After the local function has been extracted into ADFunction, we can differentiate this function using ADIFOR or ADIC. To use ADIFOR, we first create a composition file specifying the file(s) containing ADFunction as well as a driver that calls ADFunction. Figure 6 gives an example of a composition file. Next we construct the ADIFOR script file shown in Figure 7. We use the AD_TOP keyword to specify that the function to be differentiated is defined by ADFunction. The independent variable (AD_IVARS) is $1 \times$ a and the dependent variable (AD_DVARS) is If_a. We do not expect the length of $1 x_{-}$a to exceed 100 , and so we use this value for AD_PMAX. For more information on ADIFOR script options, see the ADIFOR users manual [4]. If the filename for the ADIFOR script is ex $5 f$.adf, we can invoke ADIFOR using the following command.

\section{Adifor2.0 AD_SCRIPT=ex5f.adf}

ADIFOR normally generates code that uses arrays to store dense derivative objects, but it also capable of generating code that interfaces with the SparsLinC library [4]. This library provides support for creating, updating, and extracting dynamically allocated sparse derivative objects. Figure 8 shows an ADIFOR script for generating code using sparse derivative objects. To make it easier to distinguish between the two types of code generated by ADIFOR, we use the AD_SEP option to specify a prefix of $g \$$ for variables and functions in the dense version and a prefix of $g$ - for those in the SparsLinC version. The g\$ADFunction and g_ADFunction subroutines produced by ADIFOR are provided in Appendixes A and B, respectively.

ADIC can be invoked by the following command, where ex5.init is the initialization file in Figure 9 . See [5] for more information on ADIC options. The resultant code is provided in Appendix C.

adic $-x v-d$ gradient $-i$ ex5. init $-u$ 
subroutine FormFunction(snes, X, F, dummy)

C Declarations [omitted]

C Scatter ghost points to local vector

call DAGlobalToLocalBegin (da, X, INSERT_VALUES, localX, ierr)

call DAGlobalToLocalEnd (da, X, INSERT_VALUES, localX, ierr)

C Get pointers to vector data.

call VecGetArray (localX, $\mathbf{l} x_{-} v, 1 x_{-} i$, ierr)

call VecGetArray (localf,lf_v,lf_i,ierr)

C Get local grid boundaries

call DAGetCorners (da, xs,ys,PETSC_NULL, xm, ym,PETSC_NULL, ierr)

call DAGetGhostCorners (da,gxs, gys,PETSC_NULL, gxm, gym,

$\&$

PETSC_NULL, ierr)

C BEGIN local computation

ye $=y s+y m-1$

$x e=x s+x m-1$

C Compute function over the locally owned part of the grid

do $20 j=y s$, ye

row $=(j-g y s) * g x m+x s-g x s-1$

do $10 i=x s, x e$

row $=$ row +1

if ( $i$.eq. 0 .or. $j$.eq. 0

\& .or. $i$.eq. $m x-1$.or. $j$.eq. $m y-1)$ then $1 f_{-} a($ row $)=1 x_{-} a($ row $)$ else

$u=1 x_{-} a($ row $)$

$\mathrm{uxx}=$ hydhx $*$ (two*u

$\& \quad-1 x_{-} a($ row -1$)$

\& $\quad-1 x_{-} a($ row +1$\left.)\right)$

uyy $=$ hxdhy $*$ (two*u

\& $\quad-1 x_{-} a($ row $-g x m)$

\& $\quad-1 x_{-} a($ row + gxm $\left.)\right)$

lf_a $a$ row $)=u x x+$ uyy $-s c * \exp (u)$

endif

10 continue

20 continue

C END local computation

C Restore vectors

call VecRestoreArray (localX, $1 x_{-} v, 1 x_{-} i$, ierr)

call VecRestoreArray (localf,lf_v,lf_i,ierr)

c Insert values into global vector

call DALocalToGlobal (da, localF, INSERT_VALUES, F, ierr)

call PLogFlops (11*ym*xm,ierr)

return

Figure 2: FormFunction 


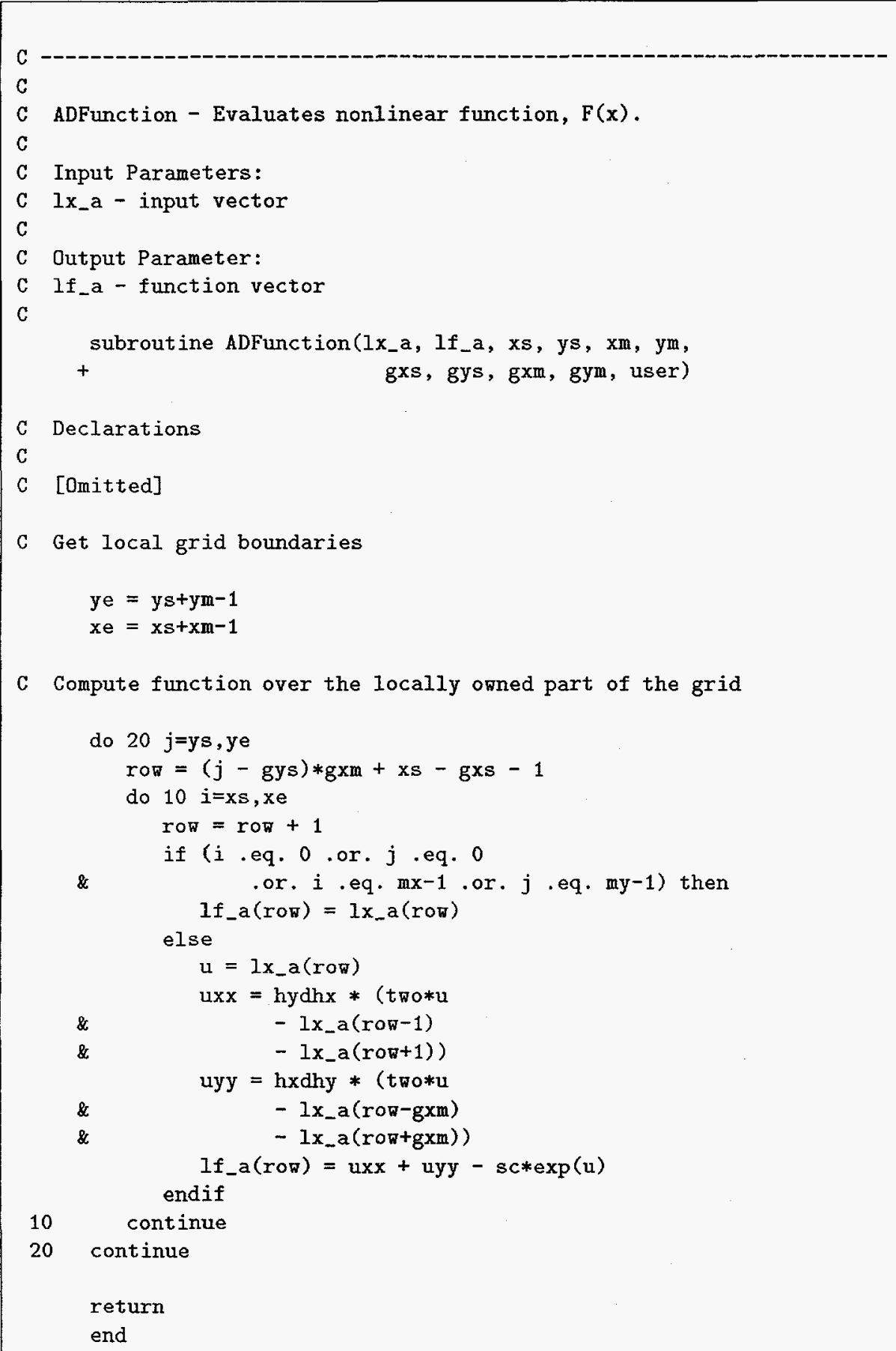

Figure 3: ADFunction 
C Scatter ghost points to local vector

call DAGlobalToLocalBegin (da, X, INSERT_VALUES, localX, ierr)

call DAGlobalToLocalEnd (da, X, INSERT_VALUES, localX, ierr)

C Get pointers to vector data.

call VecGetArray (localX, lx_v, lx_i, ierr)

call VecGetArray (localF,lf_v,lf_i, ierr)

C Get local grid boundaries

call DAGetCorners (da, xs,ys,PETSC_NULL, $x \mathrm{~m}, \mathrm{ym}$, PETSC_NULL, ierr)

call DAGetGhostCorners(da,gxs,gys,PETSC_NULL,gxm,gym,

$\&$

PETSG_NULL, ierr)

Figure 4: PETSc Predeclaration

C Restore vectors

call VecRestoreArray (localX, $1 x_{-} v, 1 x_{-} i$, ierr)

call VecRestoreArray (localF, If_v, If_i,ierr)

C Insert values into global vector

call DALocalToGlobal (da,localF, INSERT_VALUES, F, ierr)

call PLogFlops (11*ym*xm, ierr)

Figure 5: PETSc Postdeclaration

$\operatorname{ex} 5 f . f$

Figure 6: ADIFOR composition file

Figure 7: ADIFOR script 


$\begin{array}{ll} & \\ \text { AD_TOP } & =\text { ADFunction } \\ \text { AD_PMAX } & =100 \\ \text { AD_IVARS } & =1 \mathrm{x} \text { a } \\ \text { AD_DVARS } & =1 \mathrm{f} \text {-a } \\ \text { AD_PRDG } & =\text { sprse5.cmp } \\ \text { AD_FLAVOR } & =\text { sparse } \\ \text { AD_SEP } & =- \\ \text { AD_OUTPUT_DIR } & =.\end{array}$

Figure 8: ADIFOR script specifying use of SPARSLinC sparse linear algebra routines

[SOURCE_FILES]

func5.c

Figure 9: ADIC initialization file

\section{Constructing FormJacobian}

We constructed four versions of FormJacobian. The first version uses the ADIFOR-generated routine g\$ADFuction to compute the Jacobian. This version is most appropriate when the Jacobian is dense. The second version uses the ADIFOR-generated routine g_ADFunction to compute a sparse representation of the Jacobian. This version is most appropriate when the Jacobian is sparse but the sparsity pattern is unknown. The third version employs $g \$ A D F u n c t i o n$ to compute a compressed representation of the Jacobian using the strategy discussed in [1]. This method uses a coloring of the Jacobian to store structurally othogonal columns of the Jacobian in a single column of the compressed representation. The structure of the Jacobian can be supplied by the user or determined automatically. The final version uses the ADIC-generated routine ad_ADFunction to compute the Jacobian.

As enumerated earlier, the FormJacobian routine consists of five parts: PETSc predeclarations, AD setup, calling of the AD-generated function, reformatting of the Jacobian, and PETSc postdeclarations. The first and fifth parts are based on the FormFunction routine and are the same for all versions. In the following sections we describe the other three phases for each version. See Appendixes D-G to see how the five phases are assembled into a complete subroutine.

\subsection{Jacobian}

In this version, we evaluate the Jacobian matrix over the locally owned part of the grid by calling the dense version of ADIFOR-generated code. Here we present the three phases of AD setup, calling the AD-generated routine, and transferring data to PETSc data structures. The complete FormJacobian routine can be found in Appendix D.

\subsubsection{AD Setup}

The following variables are declared for use in AD.

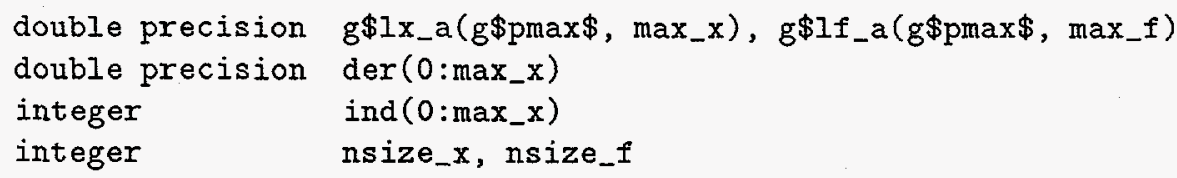


The parameter $g \$ p$ max $\$$ is the maximum number of columns in the Jacobian. Typically this will be the same as max_x, the length of $1 x_{-} a$, and should be consistent with the value used for AD_PMAX in the ADIFOR script. The variables $g \$ 1 x_{-} a$ and $g \$ 1 f_{-} a$ are used to store the seed matrix (see [4] for more information on seed matrices) and Jacobian, respectively. The variables der and ind are used in transferring the Jacobian to a PETSc matrix. The variables nsize $x$ and nsize $f$ are used to store the size of the local portions of $X$ and $F$, respectively.

To compute the Jacobian, we initialize the seed matrix $g \$ 1 x_{-} a$ to the identity matrix. In order to do so, we need to know the size of the local portion of vector $\mathrm{X}$, which can be determined by using the following PETSc call.

call VecGetLocalSize(localX,nsize_x, ierr)

Once the size of localx is known, we perform the following initialization.

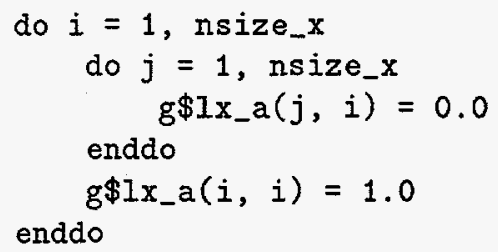

\subsubsection{Call g\$ADFunction}

We invoke the $\mathrm{AD}$-generated routine as follows.

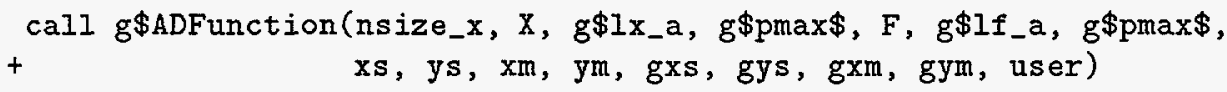

\subsubsection{Transfer AD-generated Jacobian into PETSc Data Structures}

To assemble global stiffness matrices, one needs to be able to determine the global node number of each local node including the ghost nodes. These values can be determined by using the PETSc routine DAGetGlobalIndices.

call DAGetGlobalIndices (da,n, ltog_v,ierr)

The output argument $\mathrm{n}$ contains the number of local nodes, including ghost nodes, while 1tog_v contains a list of the global indices that correspond to the local nodes.

We determine the size of the local portion of $F$ using the same PETSc routine as we did for $\mathrm{X}$.

call VecGetLocalSize(IocalF,nsize_f,ierr)

We store derivatives in the PETSc Jacobian matrix row by row as follows. The expressions 1 tog_v $(j-1)$ and 1 tog_v $(i-1)$ arise because indices in PETSc start at 0 , as in $C$, while the variables we have used in $\mathrm{AD}$ follow the Fortran convention of starting at 1.

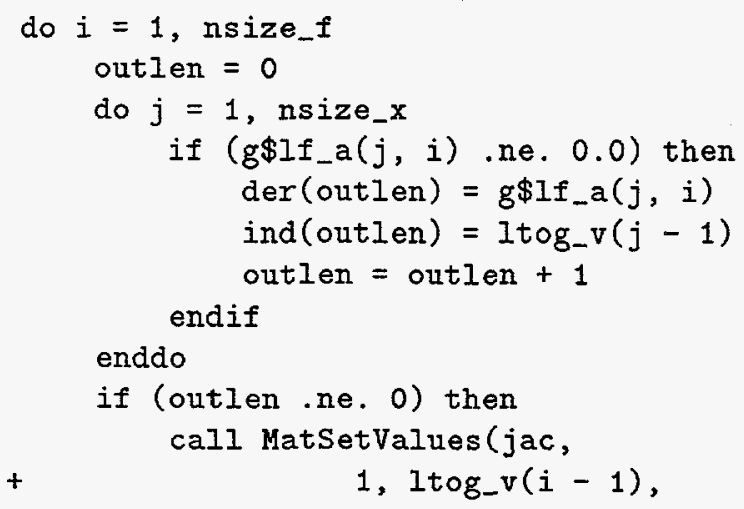




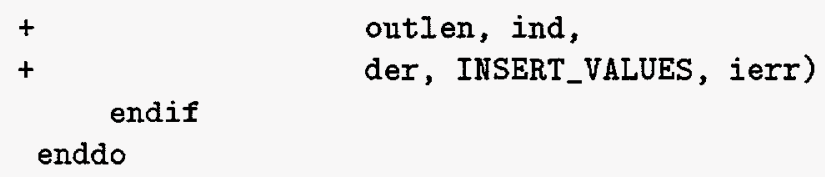

\subsection{Sparse Jacobian}

In the second version, we evaluate the Jacobian matrix over the locally owned part of the grid by calling the SparsLinC version of ADIFOR-generated code. Here we present the three phases of AD setup, calling the AD-generated routine, and transferring data to PETSc data structures. The complete FormJacobian routine can be found in Appendix $\mathrm{E}$.

\subsubsection{AD Setup}

In order to use SparsLinC, we must initialize it using the following call.

$$
\text { call xspini }
$$

After we have finished using SparsLinC, we should clean up with the following call.

$$
\text { call xspfra }
$$

The calls can be performed within the FormJacobian routine, or at a higher level, such as the main program. If FormJacobian is to be used more than once, we can reduce the overhead of calling these routines by calling them from the main program.

We declare the ADIFOR derivative variables as follows.

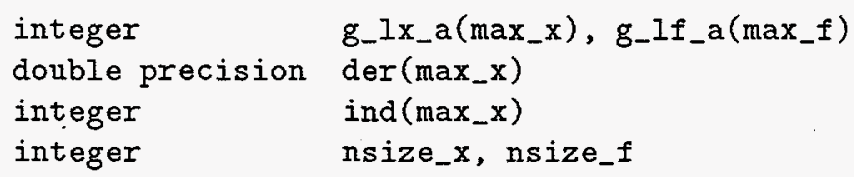

These variables play the same roles as they did in the dense version. Note, however, that g\$1x_a and g\$1f_a do not have a leading dimension of $\mathrm{g} \$$ pmax $\$$ as in the dense case. This is because these variables are used as pointers to dynamically allocated SparsLinC data structures.

Again we initialize the seed matrix to the identity matrix. However, since sparse derivative vectors are being used, each seed vector $g_{-} 1 x_{-} a(i)$ contains only a single nonzero entry, with index $i$ and value one.

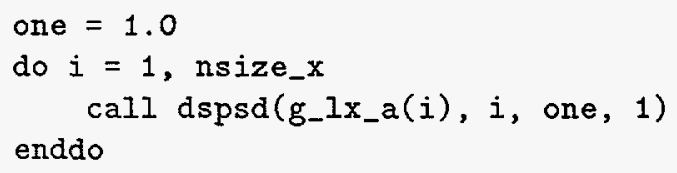

\subsubsection{Call g_ADFunction}

We invoke the $\mathrm{AD}$-generated routine as follows.

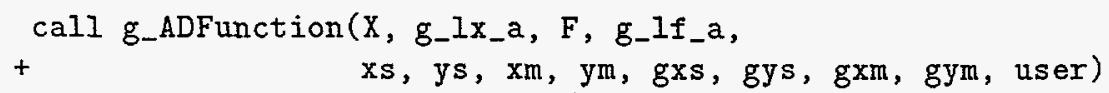

\subsubsection{Transfer AD-generated Jacobian into PETSc Data Structures}

We store derivatives in the PETSc Jacobian matrix row by row as follows. The dspxsq routine extracts derivative values from the SparsLinC data structure g_lf_a(i) and stores the indices and values in ind and der, respectively. 


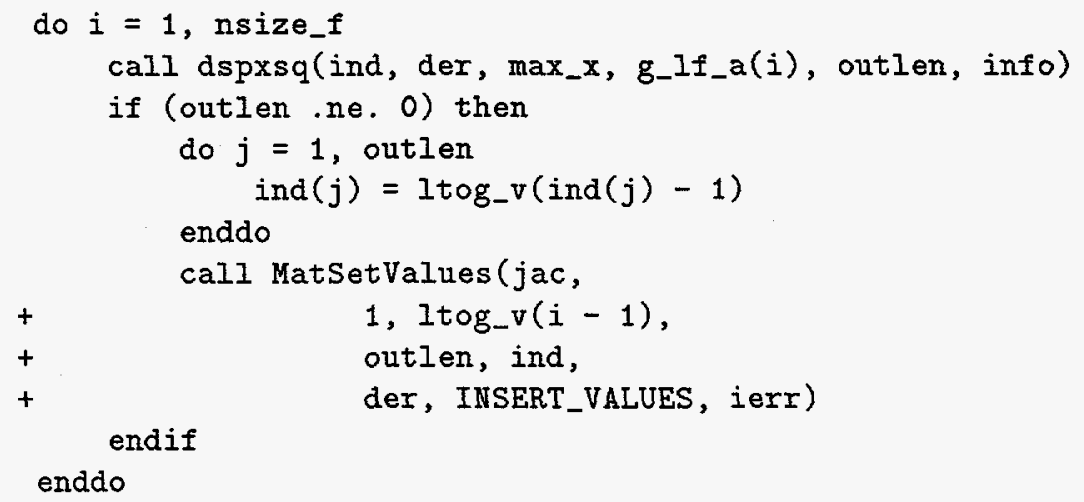

\subsection{Compressed Jacobian}

In the third version, we compute a compressed Jacobian matrix over the locally owned part of the grid by calling the ADIFOR-generated code. Our basic strategy is to compute a sparse Jacobian using SparsLinC the first time the Jacobian is computed, then use the sparsity pattern of this Jacobian to identify structurally orthogonal columns of the Jacobian. Thereafter, we compute a compressed Jacobian and test to verify that the sparsity pattern has not changed. If the pattern has changed, we recompute the Jacobian using SparsLinC. Because this scheme is complex, we encapsulate it in d_ADFunction, a routine called by FormJacobian. The complete FormJacobian and d_ADFunction routines can be found in Appendix F.

\subsubsection{AD Setup}

We declare the following derivatives for use in $\mathrm{AD}$.

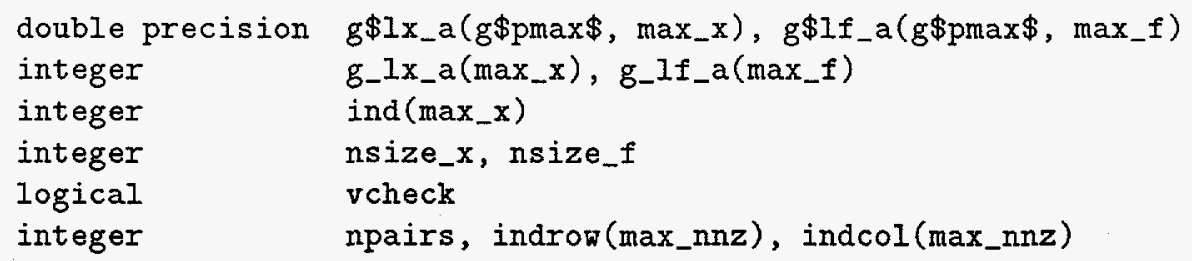

The variable $\mathrm{g} \$ 1 \mathrm{x}_{-} \mathrm{a}$ is used to store a seed matrix based on the sparsity pattern of the Jacobian, while $g \$ 1 f_{-} a$ is used to store the compressed Jacobian. The parameter $\mathbf{g} \$$ pmax $\$$ should be at least the number of columns in the compressed Jacobian. A conservative value is the number of columns in the Jacobian. The variables $g_{-} I x_{-} a$ and $g_{-} l f_{-} a$ are pointers to sparse representations of the seed matrix and Jacobian, respectively. The flag vcheck indicates whether we should check whether the sparsity structure of the Jacobian has changed. The variables npairs, indrow, and indcol are used to store the sparsity structure. The variable ind is used in transferring the Jacobian to the PETSc matrix.

Because we use SparsLinC for the first Jacobian evaluation, we must perform the same initialization and cleanup as in the sparse version. In the event that the sparsity pattern is already known, the user can supply it using the variables npairs, indrow, and indcol and the first evaluation can be performed by using a compressed Jacobian rather than SparsLinC.

In computing the compressed Jacobian, we call the dsm coloring routine to identify sets of structurally orthogonal columns. The "colors" of the columns are stored in ngrp, and the number of colors is stored in maxgrp. We compress the Jacobian by storing all columns of the same color in the same column of $g \$ 1 f_{-} a$. We accomplish this by initializing the seed matrix $g \$ 1 x_{-} a$ as follows.

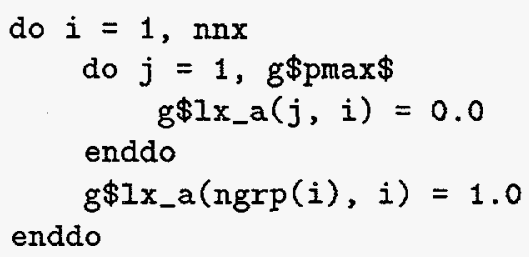


If we wish to verify that the sparsity structure has not changed, we add a random column to the seed matrix. The compressed Jacobian will contain a Jacobian-vector product in this column, which can be used to check whether the sparsity pattern has changed.

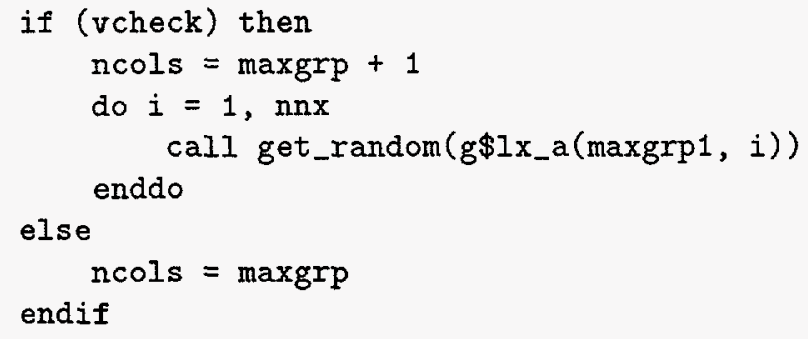

\subsubsection{Call $g \$ A D F u n c t i o n$}

We invoke the AD-generated routine as follows.

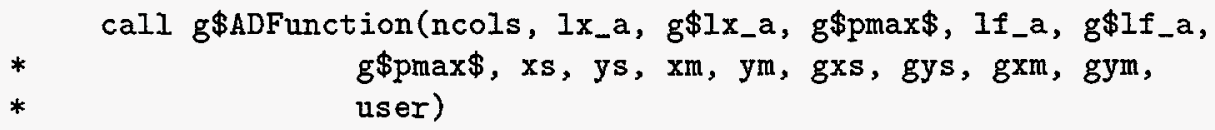

\subsubsection{Determine Whether Sparsity Pattern Has Changed}

If vcheck is true, we compare the Jacobian-vector product computed in the last column of $g \$ 1 f_{-} a$ with the product of the Jacobian stored in the rest of $g \$ 1 f_{-}$a (in compressed form) with the random vector in the last column $g \$ 1 x_{-} a$. If they are the same, we believe that the current sparsity pattern is still valid, and we accept the derivatives. If not, we reevaluate the Jacobian using SparsLinC as described above.

After recomputing the Jacobian, we compare the sparsity patterns. If the difference between these sparsity patterns is "small", then we merge (by union) the two index sets. Otherwise, we discard the old one and replace it with the new sparsity pattern. See Appendix $F$ for the actual implementation of this strategy.

\subsubsection{Transfer AD-generated Jacobian into PETSc Data Structures}

Because columns of the same color are structurally orthogonal, we can recover each nonzero element of the Jacobian by using the row index and color of the column index for that element. Thus, using a rowpointer array ptr_F, a column-index array id_F, and an array the coloring information, we can construct a column-value array $d_{-} F$, such that the nonzero values for the $i-$ th row of the Jacobian are stored in $d_{-} F\left(\operatorname{ptr} r_{-} F(i)\right)-d_{-} F\left(p t r_{-} F(i+1)-1\right)$. Derivatives are then transferred into a PETSc Jacobian matrix row by row as follows.

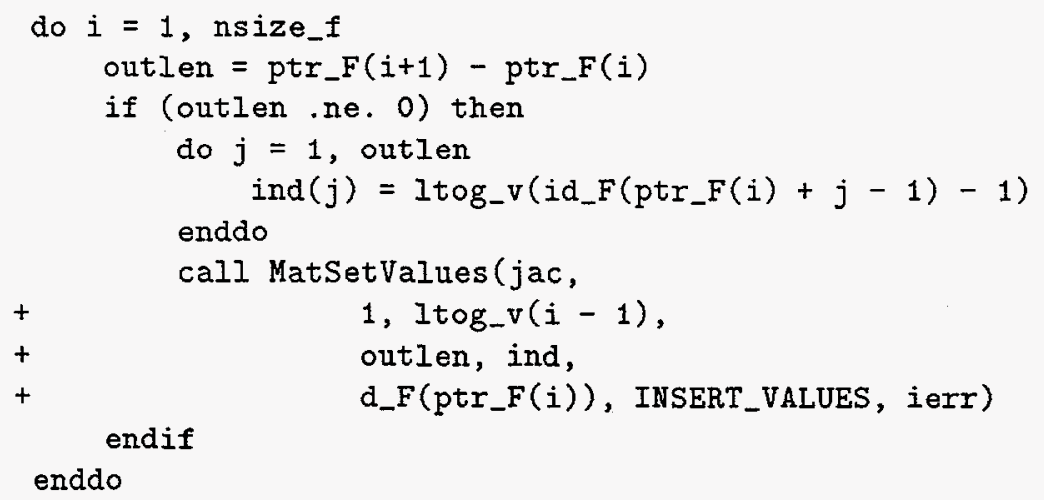




\subsection{ADIC Jacobian}

In the fourth version, we evaluate the Jacobian matrix over the locally owned part of the grid by calling the ADIC generated code. Again we present the three phases of AD setup, calling the AD-generated routine, and transferring data to PETSc data structures. The complete FormJacobian routine can be found in Appendix G.

\subsubsection{AD Setup}

We include two header files that define data structures and macros for AD.

\#include "ad_deriv.h"

\#include "adintrinsics.h"

We also declare the following variables for use in AD.

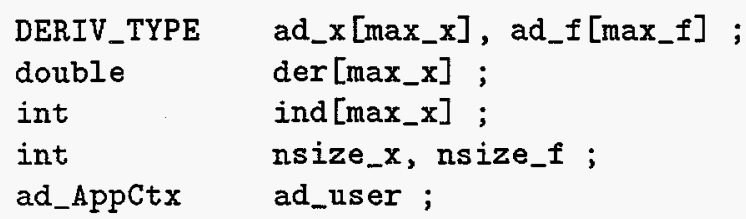

The variable ad_ $x$ is an array of data structures of type DERIV_TYPE that contain fields for both the value of $\mathbf{x}$ and the derivative vector associated with $\mathbf{x}$. Thus, this variable plays the role of the Fortran variables $\mathbf{x}_{-} \mathbf{a}$ and $g \$ 1 x_{-} a$, combined. Similarly, the variable ad_f plays the same role as $1 f_{-} a$ and $g \$ l f_{-} a$. The variables der, ind, nsize $x$, and nsize $f$ play exactly the same role as the do in the Fortran programs.

Seed matrix initalization is performed by using the following calls.

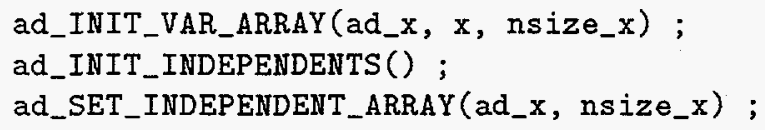

\subsubsection{Call ad_ADFunction}

We invoke the ADIC-generated routine as follows.

ad_ADFunction(ad_x, ad_f, xs, ys, xm, ym, gxs, gys, gxm, gym, \&ad_user) ;

\subsubsection{Transfer AD-generated Jacobian into PETSc Data Structures}

To assemble global stiffness matrices, one needs to be able to determine the global node number of each local node, including the ghost nodes. These values can be determined by using the PETSc routine DAGetGlobalindices.

ierr = DAGetGlobalIndices (user->da, \&nloc,\&ltog)

The output argument nloc contains the number of local nodes, including ghost nodes, while 1tog contains a list of the global indices that correspond to the local nodes.

Derivatives are extracted by using the macro ad_GET_DERIV and stored in the PETSc Jacobian matrix row by row as follows. Note the similarity between this code and the code presented for dense ADIFOR in Section 5.1.3. The principal differences arise because of the differences in matrix indexing in Fortran and C.

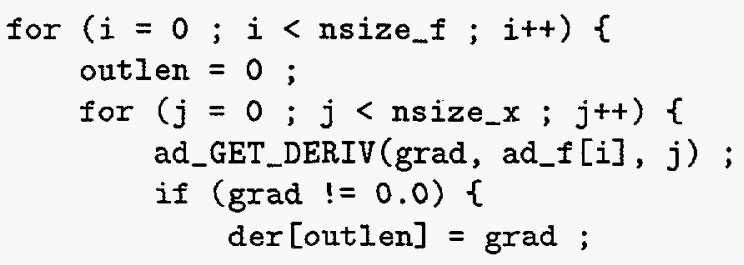




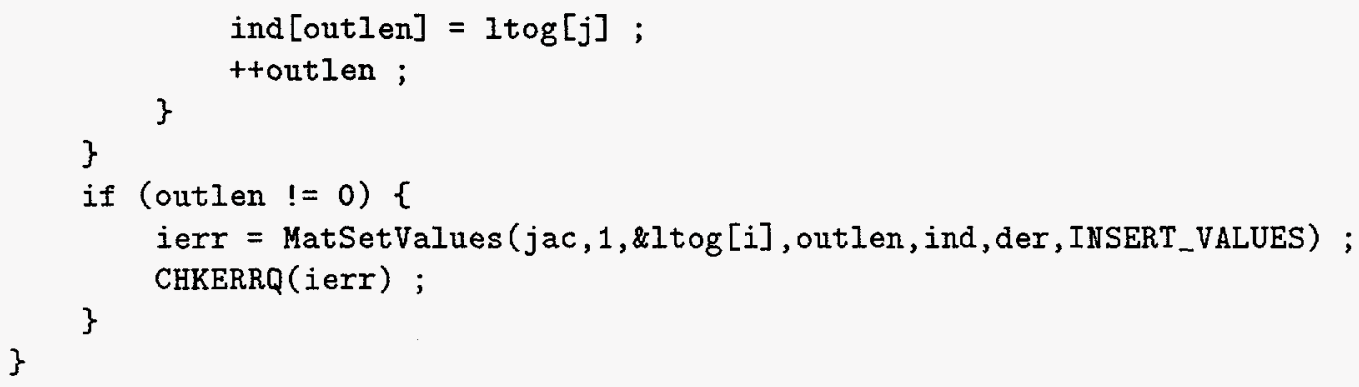

\section{Summary}

We presented a general strategy for using ADIFOR or ADIC to assist in developing a subroutine for computing the Jacobian of a function. This subroutine can be used by the SNES component of PETSc. The basic approach is to isolate a local function evaluation from PETSc data distribution instructions, apply ADIFOR or ADIC to this local function, then encapsulate the code generated using AD in a subroutine that performs the appropriate distribution of data. We demonstrated how this strategy could be applied to one of the PETSc example programs using each of four different approaches to computing the Jacobian via AD. 


\section{Appendix}

\section{A g\$ADFunction}

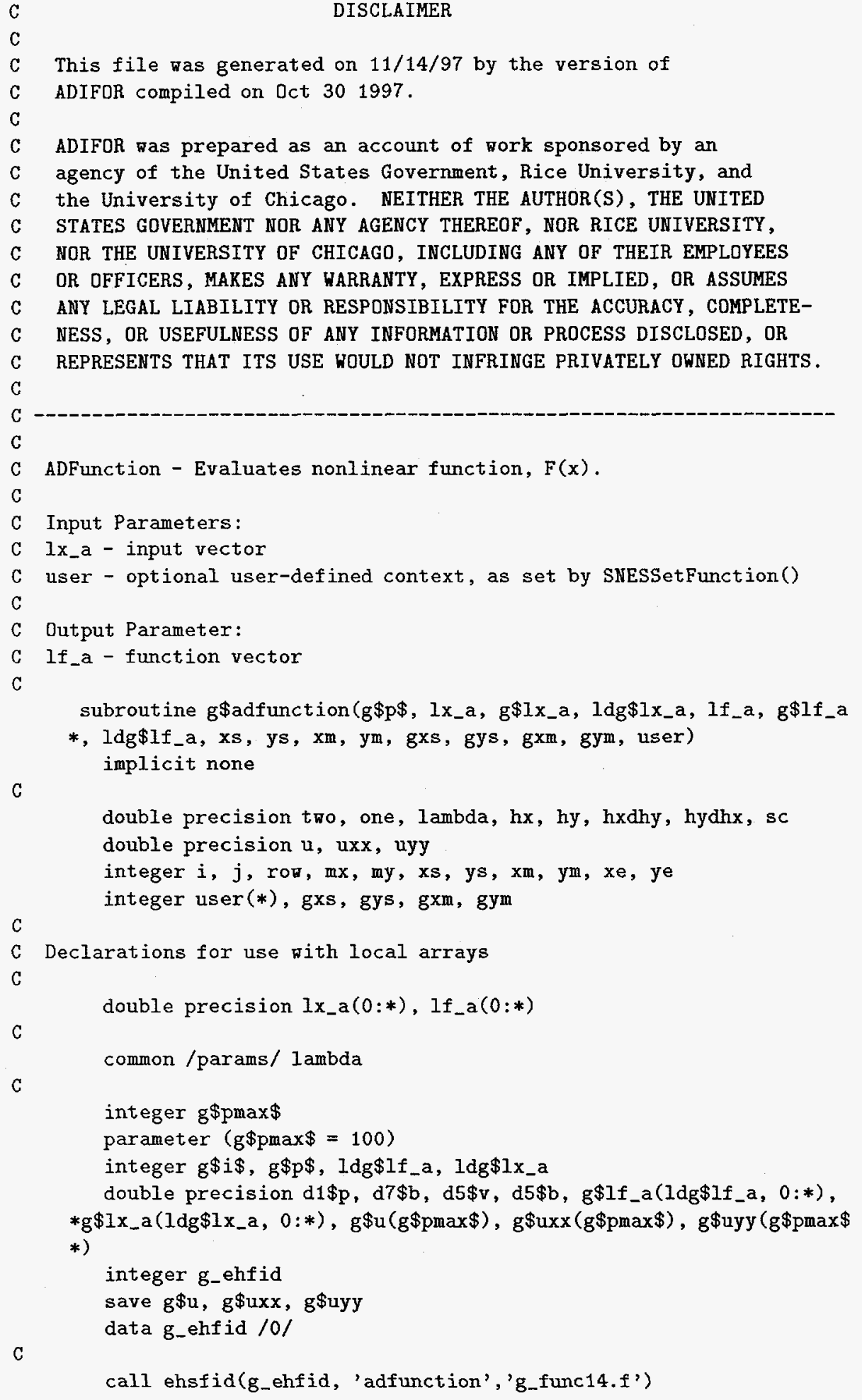




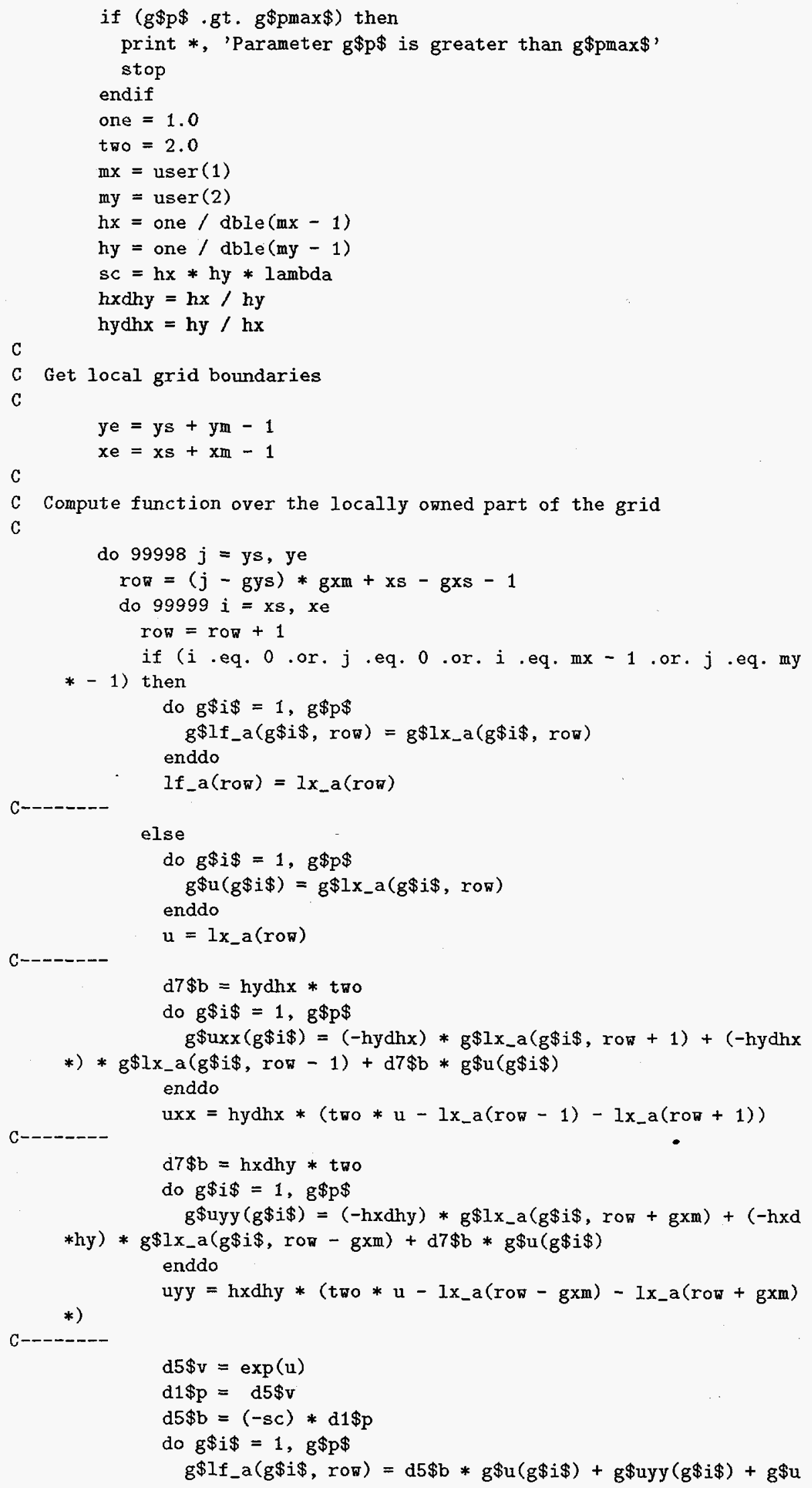




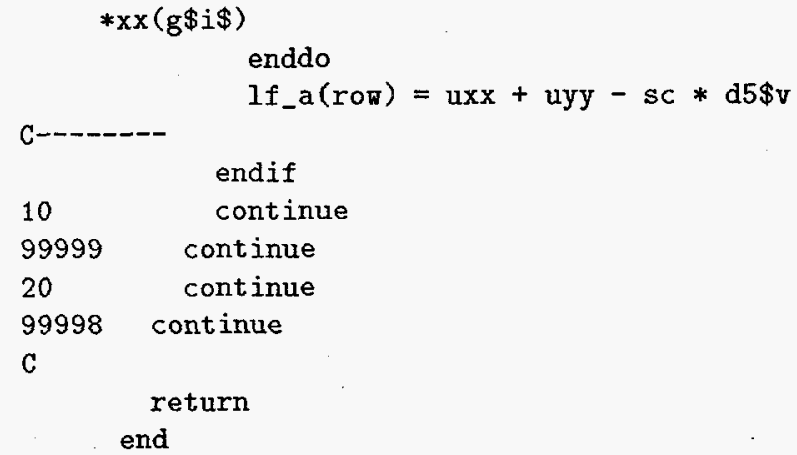

\section{B g_ADFunction}




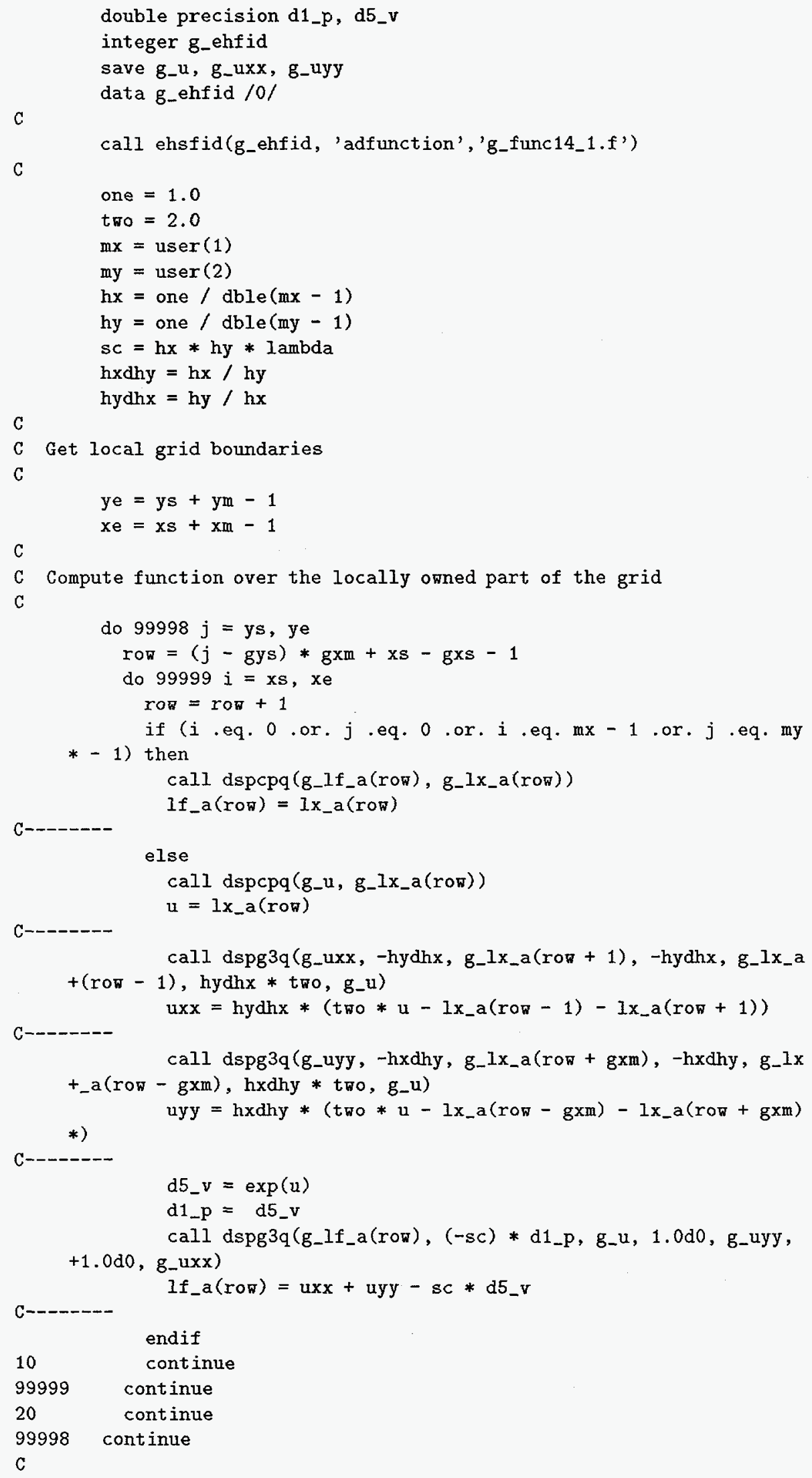


return

end

\section{C ad_ADFunction}

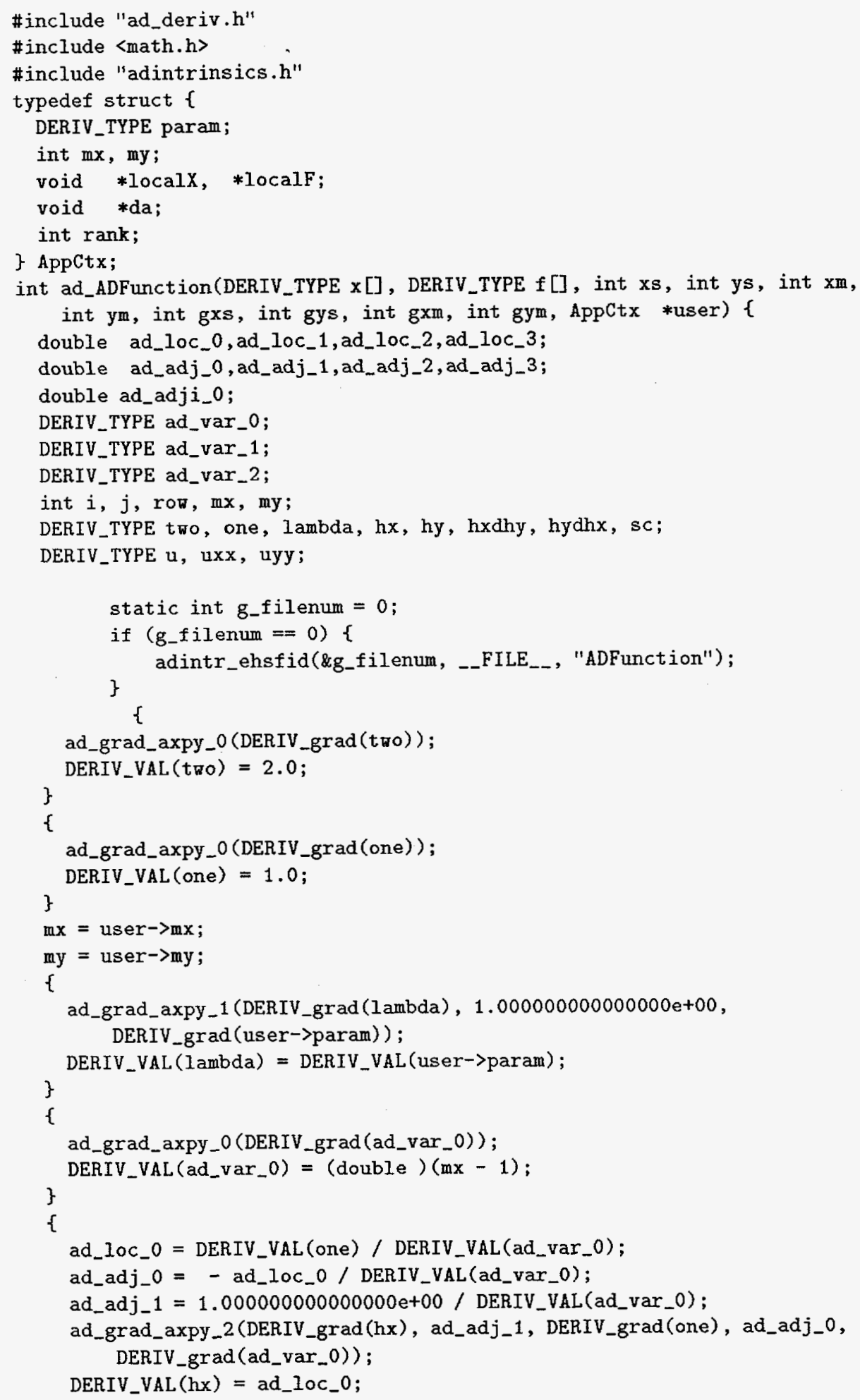


\{

ad_loc_0 = DERIV_VAL $(h x) *$ DERIV_VAL $(h y)$;

ad_loc_1 $=$ ad_loc_0 $*$ DERIV_VAL (lambda);

ad_adj_0 = DERIV_VAL $(h x) *$ DERIV_VAL (lambda);

ad_adj_1 = DERIV_VAL (hy) * DERIV_VAL (lambda);

ad_grad_axpy_3(DERIV_grad(sc), ad_adj_1, DERIV_grad $(h x)$, ad_adj_0,

DERIV_grad(hy), ad_loc_o, DERIV_grad(lambda));

DERIV_VAL $(\mathrm{SC})=\mathrm{ad}_{-} l o c_{-} 1$;

\}

t

ad_loc_0 = DERIV_VAL(hx) / DERIV_VAL(hy);

ad_adj_o $=$ - ad_loc_0 / DERIV_VAL_(hy);

ad_adj_1 $=1.000000000000000$ e $+00 /$ DERIV_VAL(hy);

ad_grad_axpy_2(DERIV_grad(hxdhy), ad_adj_1, DERIV_grad(hx), ad_adj_o, DERIV_grad (hy));

\}

DERIV_VAL (hxdhy) = ad_loc_o;

\{

ad_loc_0 = DERIV_VAL(hy) / DERIV_VAL $(h x)$;

ad_adj_o $=-$ ad_loc_0 / DERIV_VAL_hx $)$;

ad_adj_1 $=1.000000000000000 \mathrm{e}+00 /$ DERIV_VAL(hx);

ad_grad_axpy_2(DERIV_grad(hydhx), ad_adj_1, DERIV_grad(hy), ad_adj_o, DERIV_grad $(h \mathbf{x}))$;

DERIV_VAL $($ hydhx $)=$ ad_loc_0;

\}

for $(j=y s ; j<y s+y m ; j++)\{$

row $=(j-g y s) * g x m+x s-g x s-1$;

for $(i=x s ; i<x s+x m ; i++)\{$

(row) ++ ;

if $(i==0\|j==0\| i=m x-1 \| j==m y-1)\{$

\{

ad_grad_axpy_1(DERIV_grad $(f[r o w]), 1.000000000000000 e+00$,

DERIV_grad (x[row]));

\}

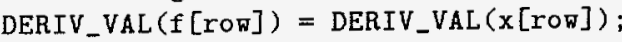

continue;

\{

\}

ad_grad_axpy_1(DERIV_grad(u), $1.000000000000000 \mathrm{e}+00$,

DERIV_grad ( $x[\operatorname{row}]))$;

DERIV_VAL $(u)=$ DERIV_VAL $(x[$ row $])$;

\}

\{

ad_loc_0 = DERIV_VAL (two) * DERIV_VAL $(u)$;

ad_IOC_1 = ad_loc_0 - DERIV_VAL $(x[$ row -1$])$; 


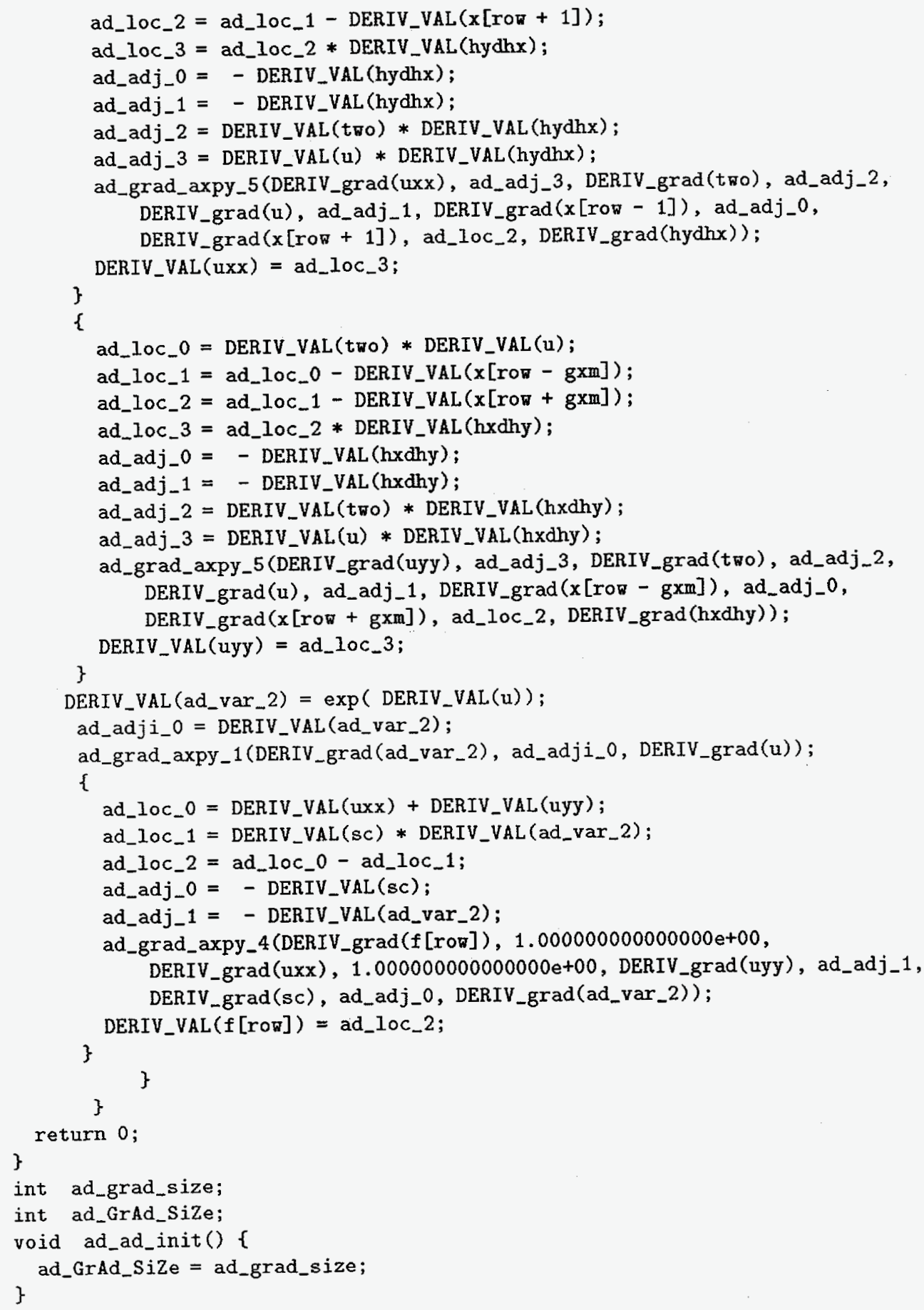

\section{Dense FormJacobian}

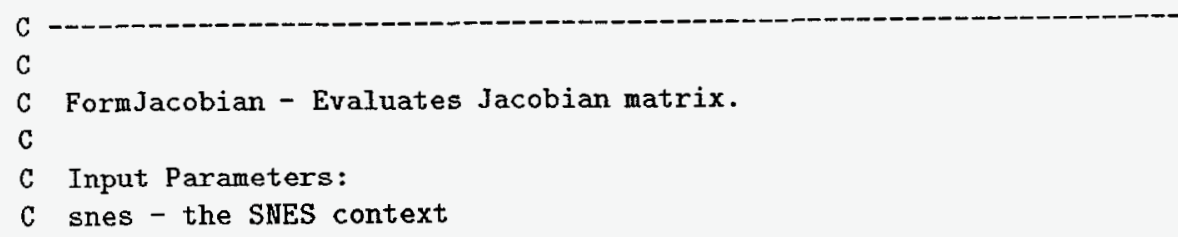




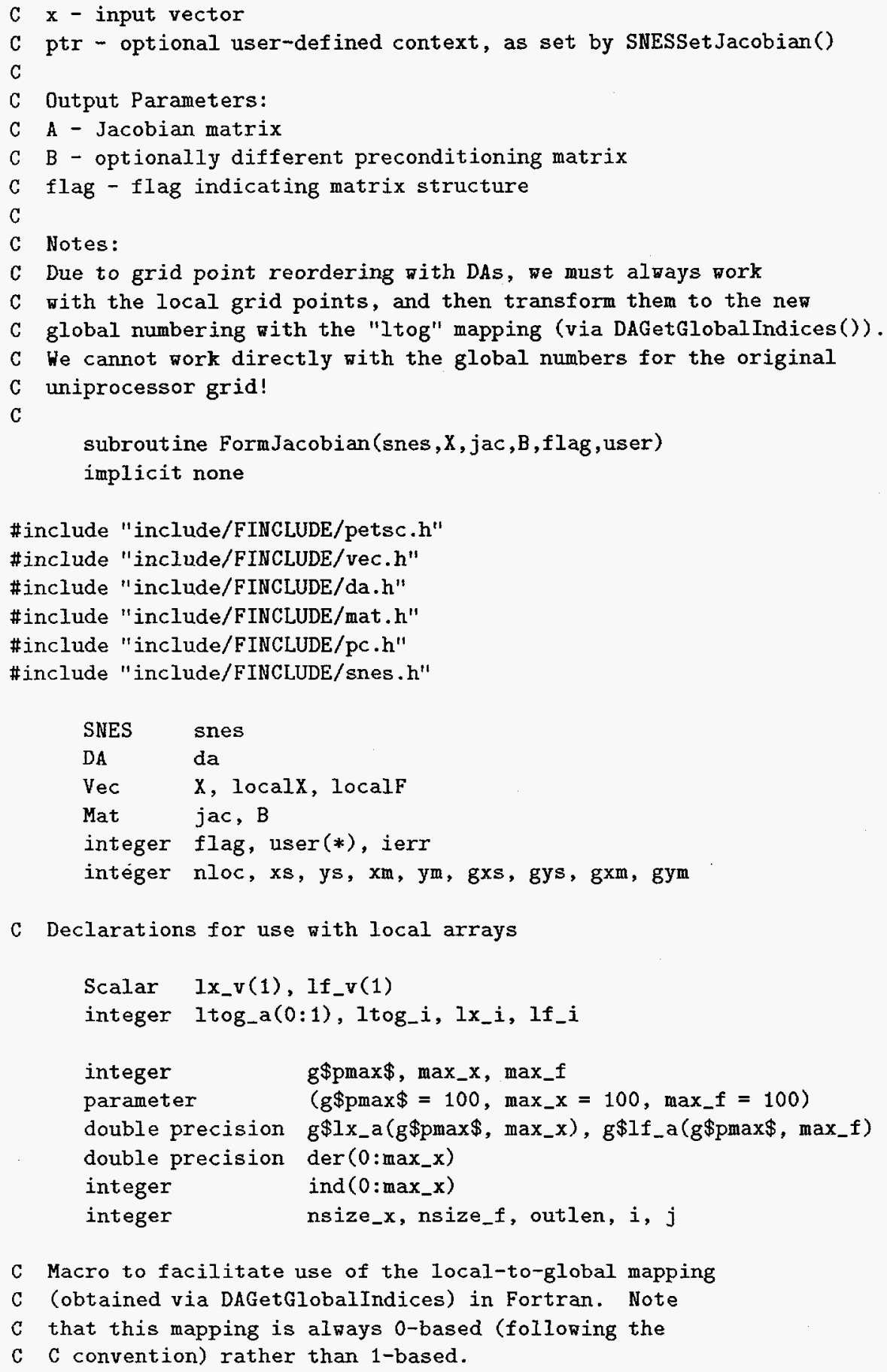

C Scatter ghost points to local vector, using the 2-step process 
C DAGlobalToLocalBegin(), DAGlobalToLocalEnd()

C Computations can be done while messages are in transition,

$C$ by placing code between these two statements.

call DAGlobalToLocalBegin (da, X, INSERT_VALUES, localX, ierr)

call DAGlobalToLocalEnd (da, X, INSERT_VALUES, localX, ierr)

C Get a pointer to vector data

call VecGetArray (1ocalX,1x_v, $1 x_{-} i$,ierr)

call VecGetArray (localF,lf_v,lf_i,ierr)

C Get local grid boundaries

call DAGetCorners (da, xs,ys,PETSC_NULL, xm, ym, PETSC_NULL, i err)

call DAGetGhostCorners (da,gxs, gys, PETSC_NULL,gxm, gym,

\& PETSC_NULL, ierr)

C Get the global node numbers for all local nodes, including ghost points

- Note that the Fortran interface to DAGetGlobalindices() differs from

C the $C$ version. See the Fortran chapter of the users manual for details.

call DAGetGlobalIndices (da,nloc,ltog_a,ltog_i,ierr)

C

call VecGetLocalsize (localX,nsize_x, ierr)

call VecGetLocalSize(localF,nsize_f,ierr)

C Initialize seed matrix \& call ADIFOR code.

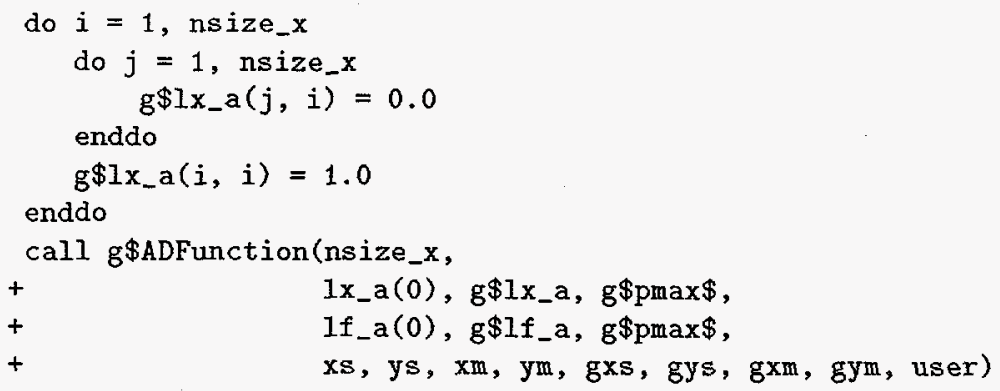

C Store derivatives into jac matrix.

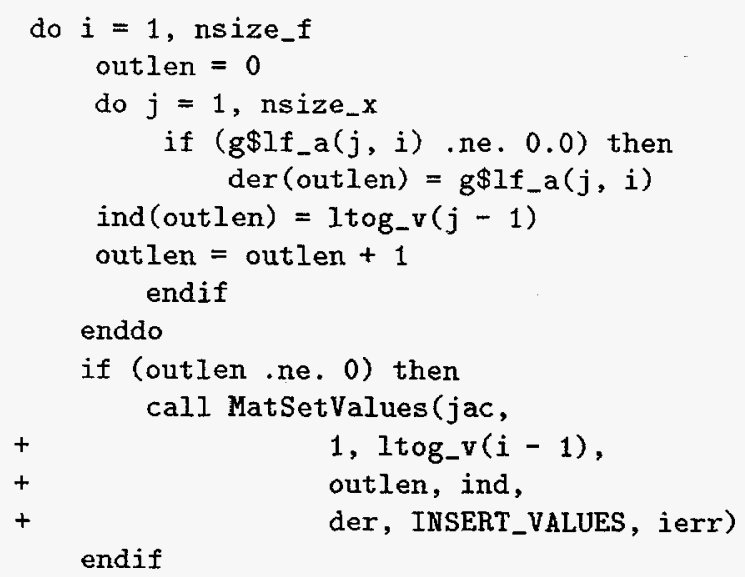


enddo

Assemble matrix, using the 2-step process:

MatAssemblyBegin(), MatAssemblyEnd()

Computations can be done while messages are in transition,

by placing code between these two statements.

call MatAssemblyBegin (jac,MAT_FINAL_ASSEMBLY,ierr)

call VecRestoreArray (localX, Ix_v, lx_i, ierr)

call MatAssemblyEnd (jac,MAT_FINAL_ASSEMBLY,ierr)

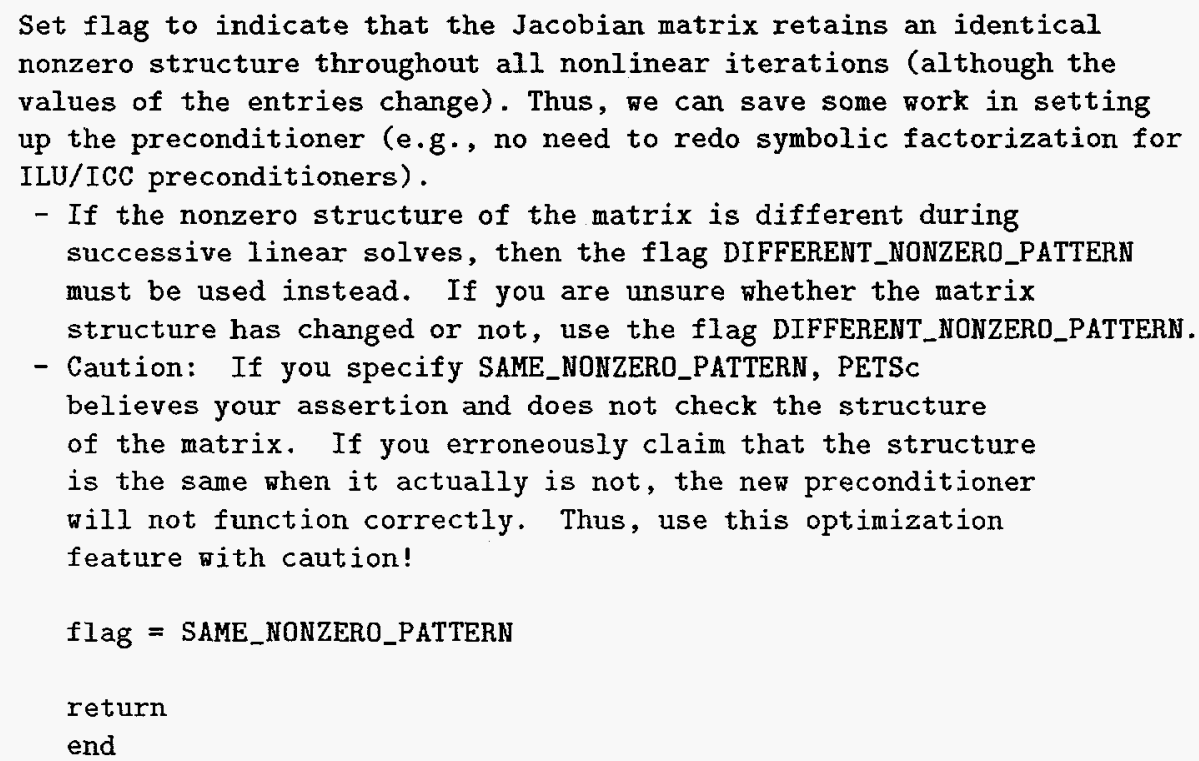

\section{E Sparse FormJacobian}

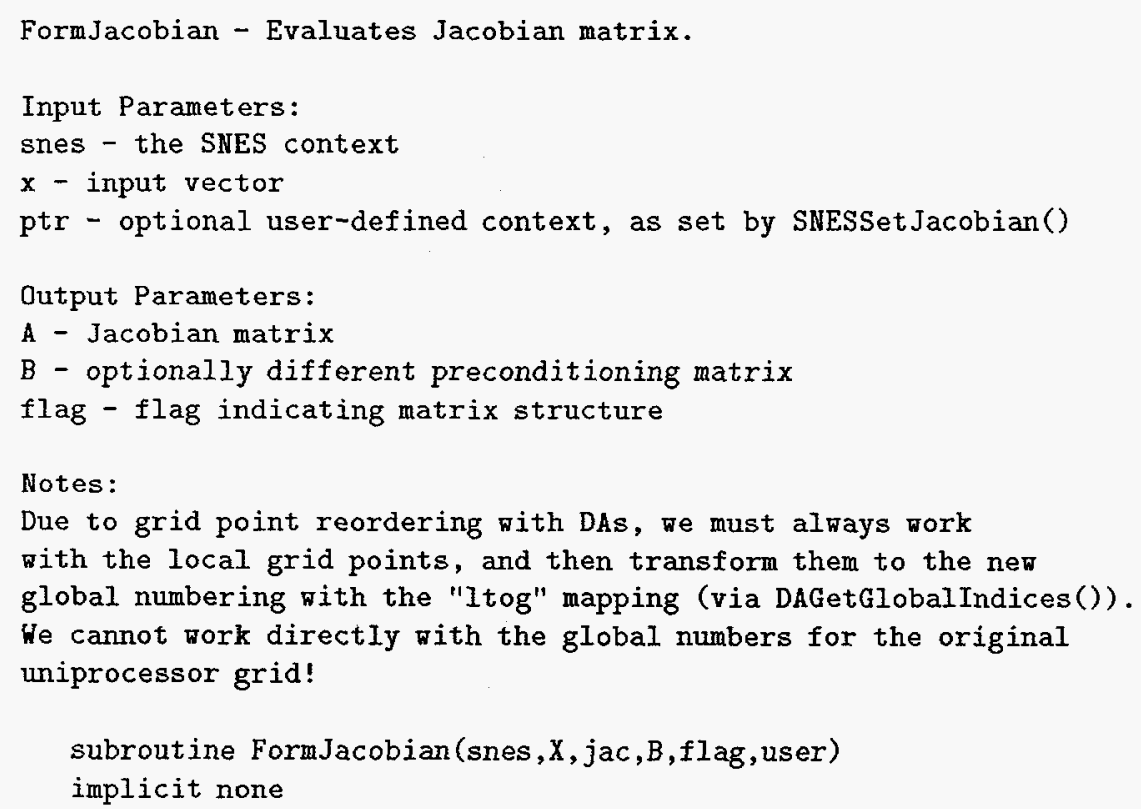


\#include "include/FINCLUDE/petsc.h"

\#include "include/FINCLUDE/vec.h"

\#include "include/FINCLUDE/da.h"

\#include "include/FINCLUDE/mat. $h$ "

\#include "include/FINCLUDE/pc. $h$ "

\#include "include/FINCLUDE/snes.h"

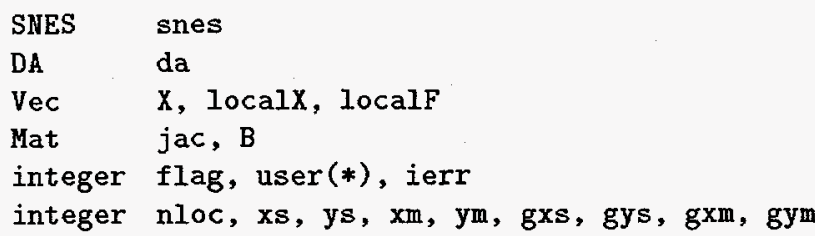

C Declarations for use with local arrays

Scalar $1 x_{-} v(1), 1 f_{-} v(1)$

integer 1 tog_a $(0: 1), 1 \operatorname{tog}_{-} i, 1 x_{-} i, 1 f_{-} i$

integer $\quad \max _{-} x, \max _{-} f$

parameter (max_x $\left.=100, \max _{-} f=100\right)$

integer $\quad g_{-} l x_{-} a\left(\max x_{-}\right), g_{-} l f_{-} a\left(\max _{-} f\right)$

double precision der(max_x)

integer ind $\left(\max _{-} x\right)$

double precision one

integer nsize_x, nsize_f, outlen, info, $i, j$

C Macro to facilitate use of the local-to-global mapping

C (obtained via DAGetGlobalIndices) in Fortran. Note

$C$ that this mapping is always 0-based (following the

C C convention) rather than 1 -based.

\#define $1 \operatorname{tog}_{-} v(i b) \quad 1 \operatorname{tog}_{-} a(1 \operatorname{tog} i+(i b))$

c Set parameters

$$
\begin{aligned}
& \text { da }=\text { user }(3) \\
& \text { localX }=\text { user (4) } \\
& \text { localF }=\text { user (5) }
\end{aligned}
$$

C Scatter ghost points to local vector, using the 2-step process DAGlobalToLocalBegin(), DAGlobalToLocalEnd()

C Computations can be done while messages are in transition,

C by placing code between these two statements.

call DAGlobalToLocalBegin (da, X, INSERT_VALUES, localX, ierr)

call DAGlobalToLocalEnd(da,X,INSERT_VALUES, localX, i err)

C Get a pointer to vector data

call VecGetArray (localX, $1 x_{-} v, 1 x_{-} i$, ierr)

call VecGetArray (localf,lf_v,lf_i,ierr)

C Get local grid boundaries

call DAGetCorners (da, xs, ys,PETSC_NULL, xm, ym,PETSC_NULL, ierr)

call DAGetGhostCorners (da,gxs,gys,PETSC_NULL,gxm,gym, 
$C$ Get the global node numbers for all local nodes, including ghost points

- Note that the Fortran interface to DAGetGlobalindices() differs from the $C$ version. See the Fortran chapter of the users manual for details.

call DAGetGlobalIndices (da,nloc, Itog_a,ltog_i,ierr)

C

call VecGetLocalsize(localX,nsize_x,ierr)

call VecGetLocalSize(localF,nsize_f,ierr)

C Initialize seed matrix \& call ADIFOR code.

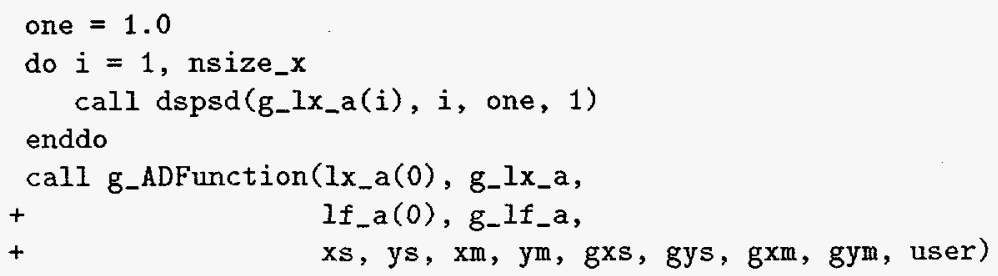

C Store derivatives into jac matrix.

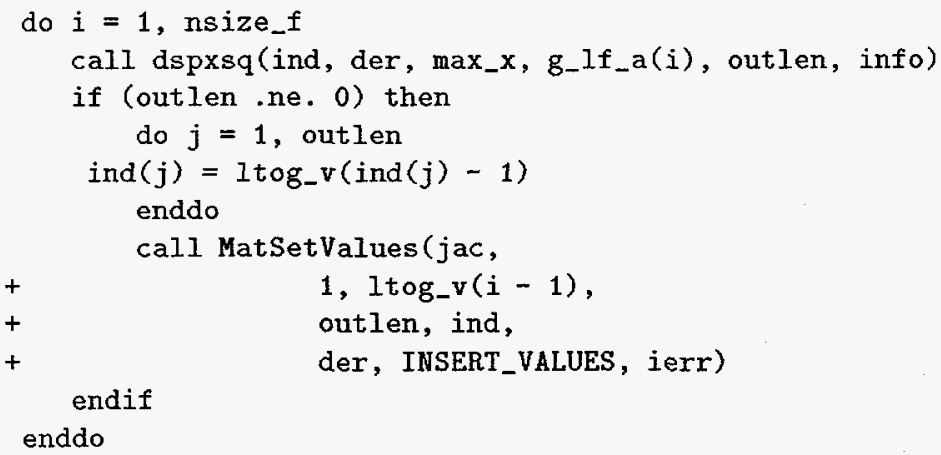

C Assemble matrix, using the 2-step process:

MatAssemblyBegin(), MatAssemblyEnd()

Computations can be done while messages are in transition,

C by placing code between these two statements.

call MatAssemblyBegin(jac,MAT_FINAL_ASSEMBLY,ierr)

call VecRestoreArray (localX, Ix_v, Ix_i, ierr)

call MatAssemblyEnd(jac,MAT_FINAL_ASSEMBLY,ierr)

\footnotetext{
Set flag to indicate that the Jacobian matrix retains an identical nonzero structure throughout all nonlinear iterations (although the values of the entries change). Thus, we can save some work in setting up the preconditioner (e.g., no need to redo symbolic factorization for ILU/ICC preconditioners).

- If the nonzero structure of the matrix is different during successive linear solves, then the flag DIFFERENT_NONZERO_PATTERN must be used instead. If you are unsure whether the matrix structure has changed or not, use the flag DIFFERENT_NONZERO_PATTERN.

- Caution: If you specify SAME_NONZERO_PATTERN, PETSc believes your assertion and does not check the structure of the matrix. If you erroneously claim that the structure
} 
flag = SAME_NDNZERO_PATTERN

return

end

\section{F Colored FormJacobian}

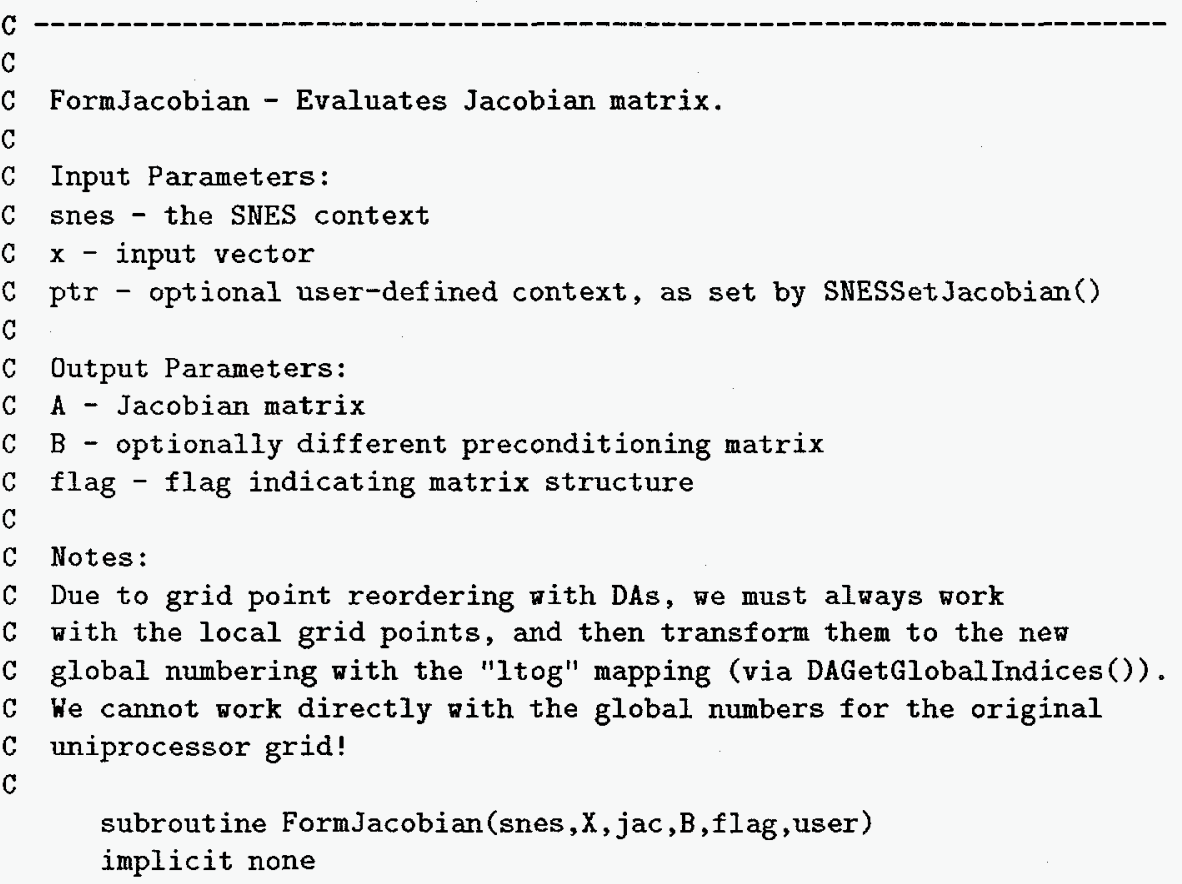




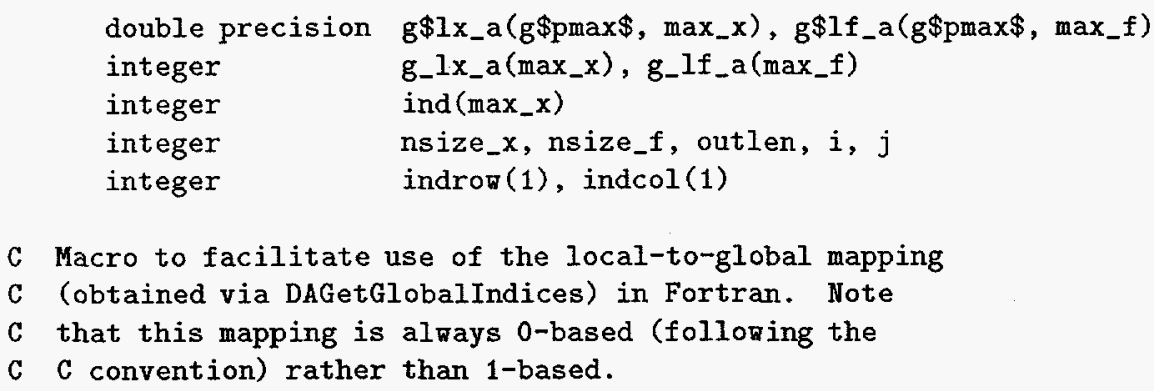

C Set parameters

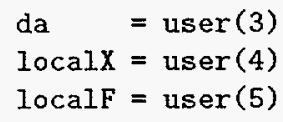

C Scatter ghost points to local vector, using the 2-step process DAGlobalToLocalBegin(), DAGlobalToLocalEnd()

Computations can be done while messages are in transition, by placing code between these two statements.

call DAGlobalToLocalBegin(da, X, INSERT_VALUES, IocalX, ierr)

call DAGlobalToLocalEnd (da, X,INSERT_VALUES, localX, ierr)

C Get a pointer to vector data

call VecGetArray (localX, lx_v,lx_i,ierr)

call VecGetArray (localf,lf_v,lf_i,ierr)

C Get local grid boundaries

call DAGetCorners (da, xs, ys,PETSC_NULL, xm,ym,PETSC_NULL, ierr)

call DAGetGhostCorners(da,gxs,gys,PETSC_NULL,gxm,gym,

\&

PETSC_NULL, ierr)

C Get the global node numbers for all local nodes, including ghost points

- Note that the Fortran interface to DAGetGlobalindices() differs from the $C$ version. See the Fortran chapter of the users manual for details.

call DAGetGlobalIndices (da,nloc,ltog_a,ltog_i,ierr)

C

call VecGetLocalsize(localx,nsize_x, ierr)

call VecGetLocalSize(localF,nsize_f,ierr)

C Initialize seed matrix \& call ADIFOR code.

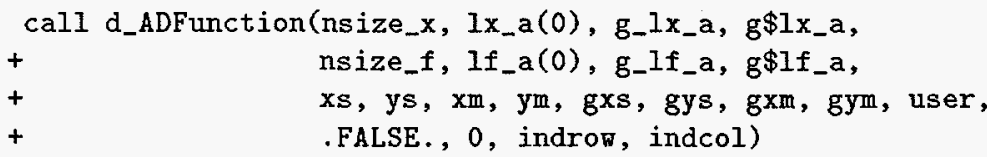

C Store derivatives into jac matrix. 


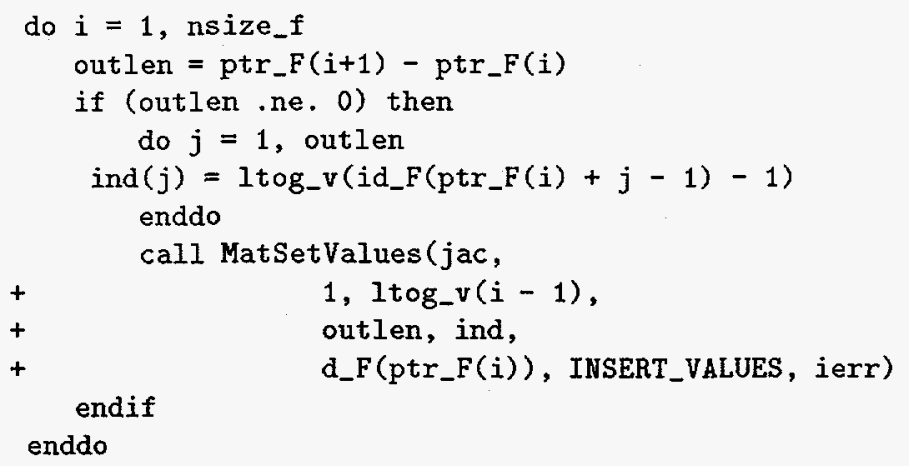

Assemble matrix, using the 2-step process:

C MatAssemblyBegin(), MatAssemblyEnd()

C Computations can be done while messages are in transition,

C by placing code between these two statements.

call MatAssemblyBegin (jac,MAT_FINAL_ASSEMBLY,ierr)

call VecRestoreArray (localX, $1 x_{-} v, 1 x_{-} i$, ierr)

call MatAssemblyEnd (jac,MAT_FINAL_ASSEMBLY, ierr)

C Set flag to indicate that the Jacobian matrix retains an identical nonzero structure throughout all nonlinear iterations (although the

values of the entries change). Thus, we can save some work in setting

up the preconditioner (e.g., no need to redo symbolic factorization for

ILU/ICC preconditioners).

- If the nonzero structure of the matrix is different during successive linear solves, then the flag DIFFERENT_NONZERO_PATTERN must be used instead. If you are unsure whether the matrix structure has changed or not, use the flag DIFFERENT_NONZERO_PATTERN.

- Caution: If you specify SAME_NONZERO_PATTERN, PETSc believes your assertion and does not check the structure of the matrix. If you erroneously claim that the structure is the same when it actually is not, the new preconditioner will not function correctly. Thus, use this optimization feature with caution!

flag = SAME_NONZERO_PATTERN

return

end

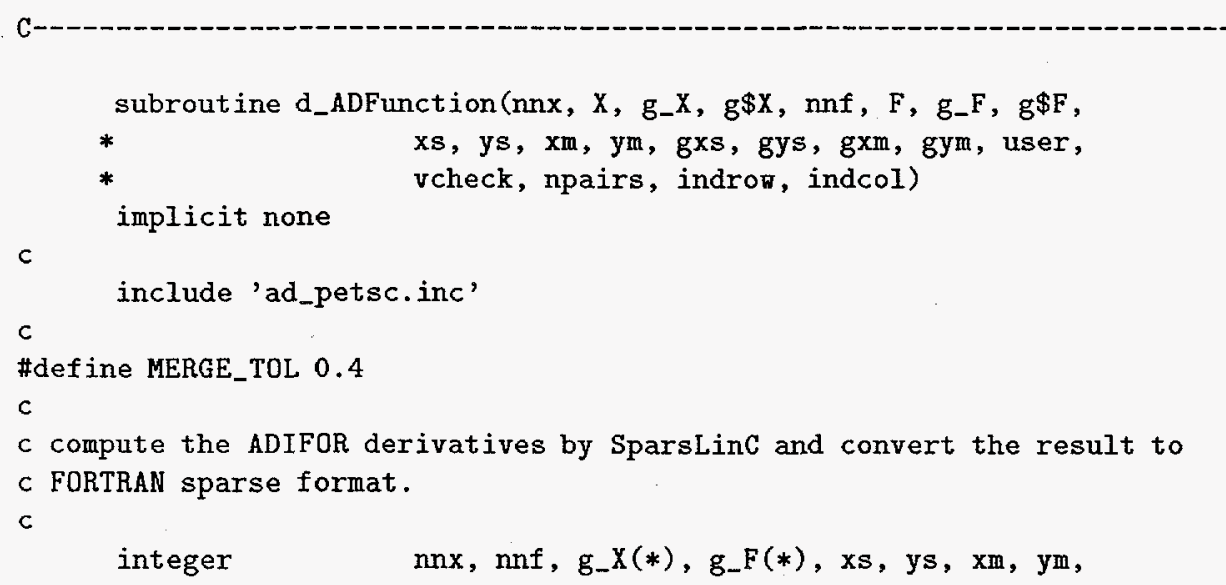




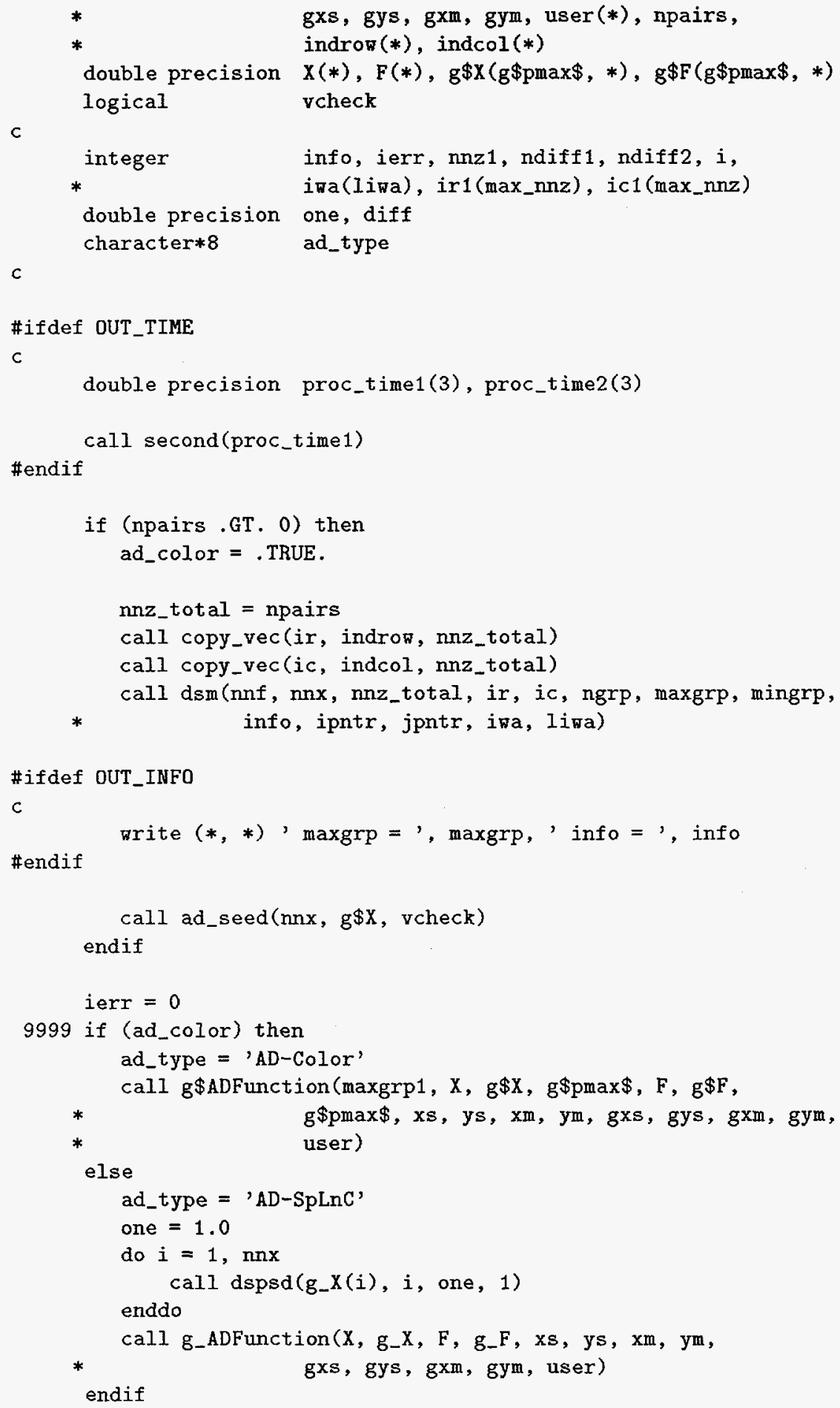




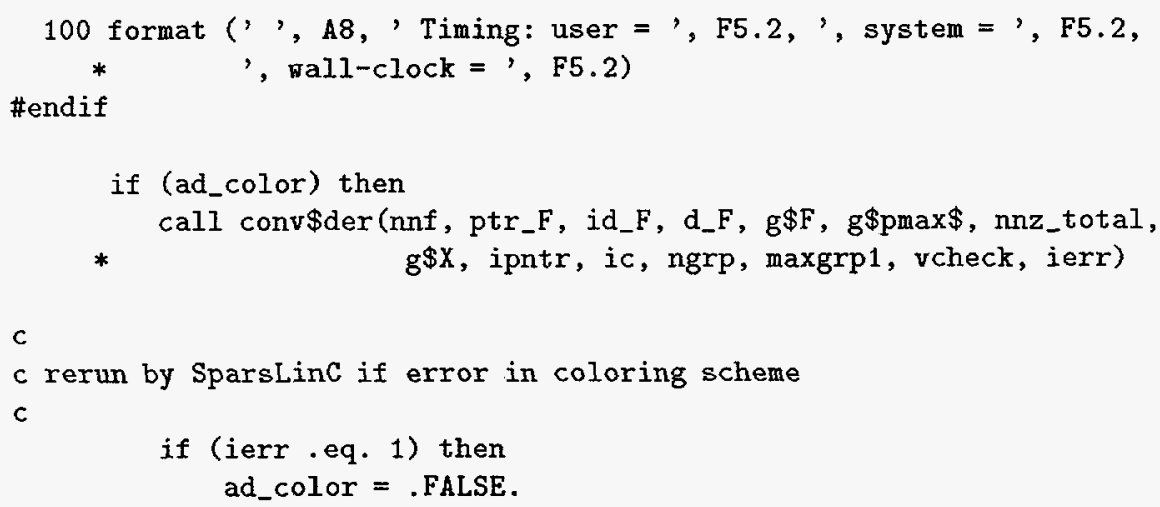




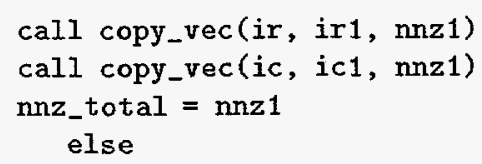




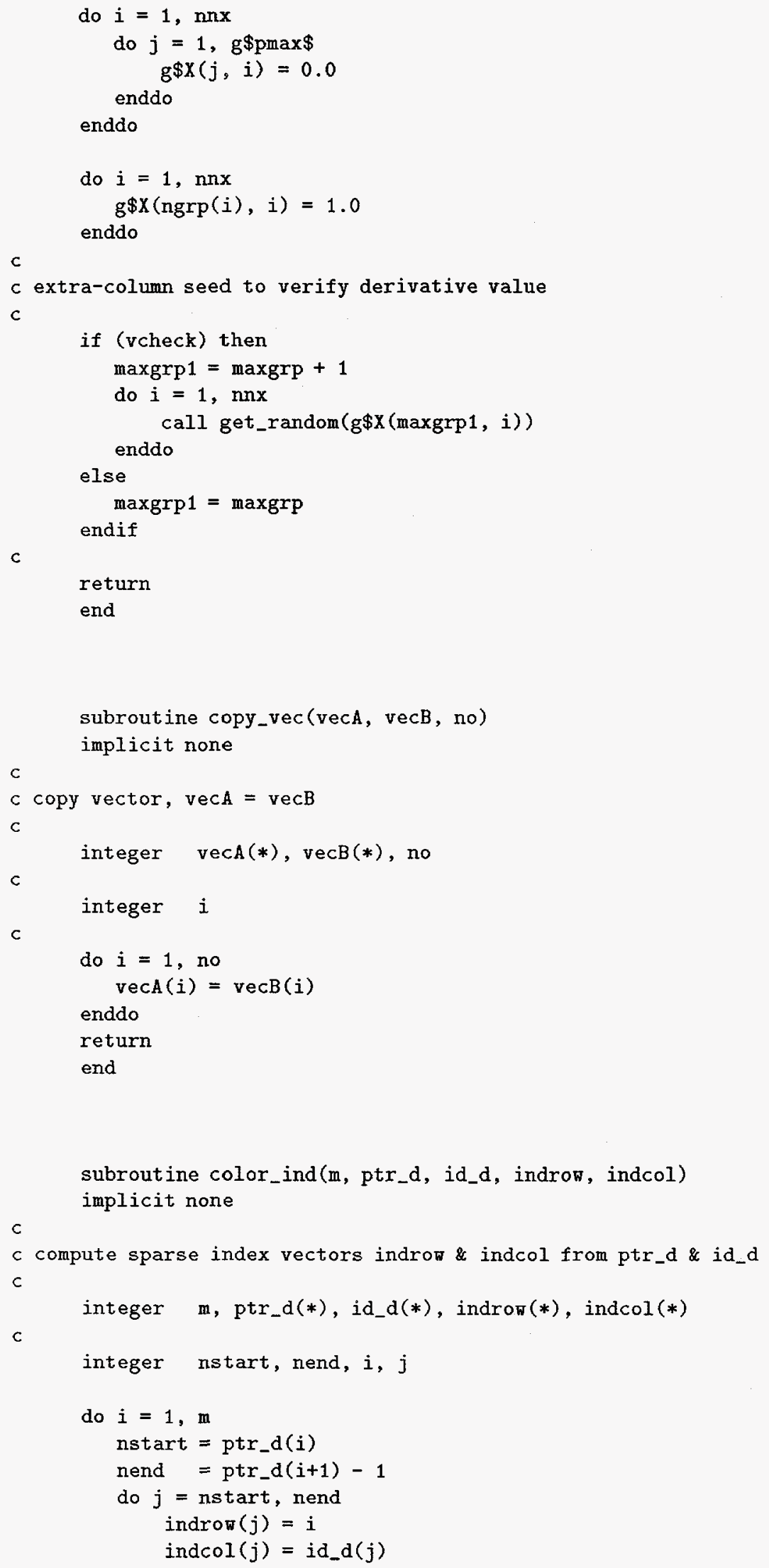




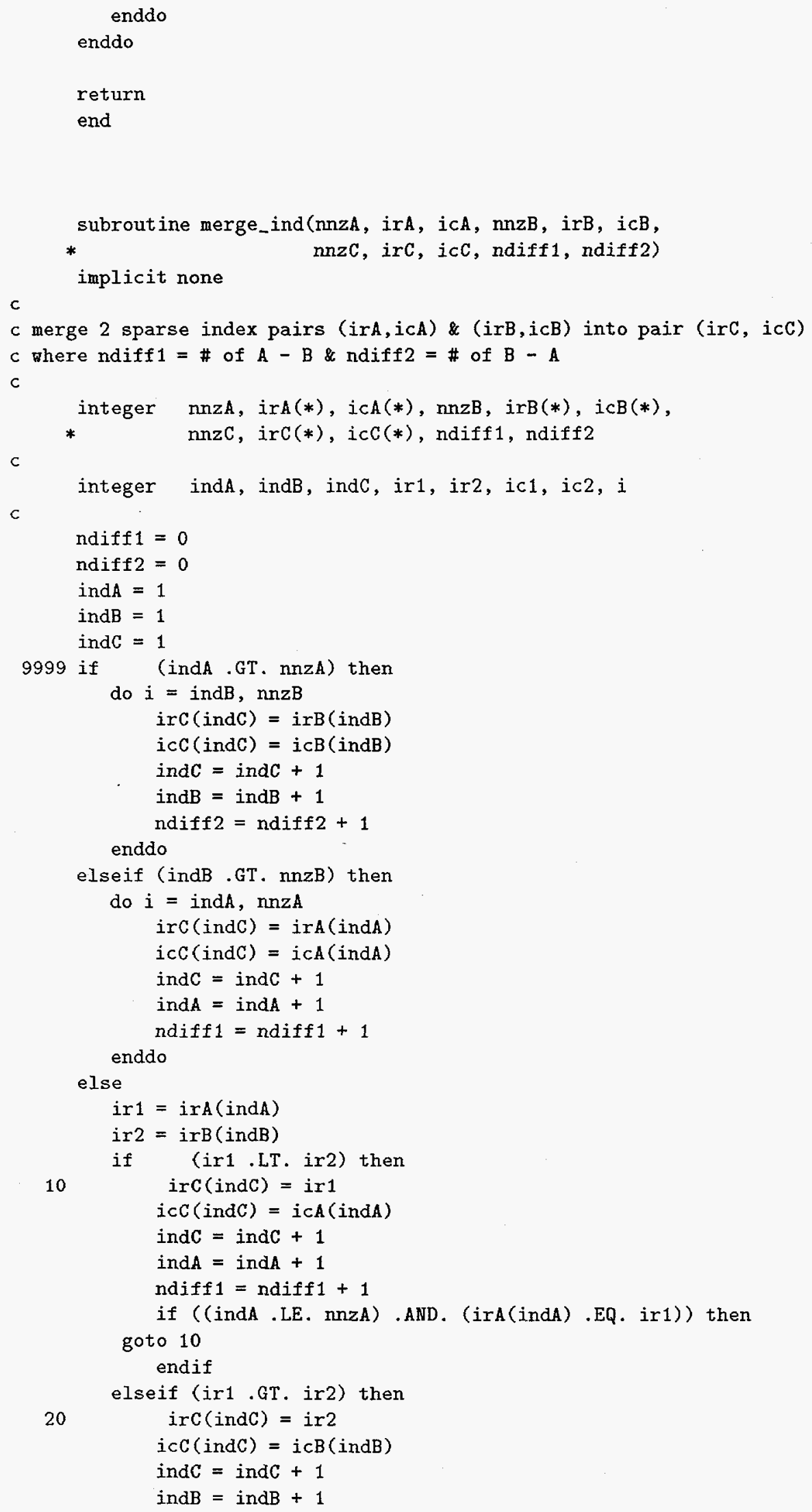




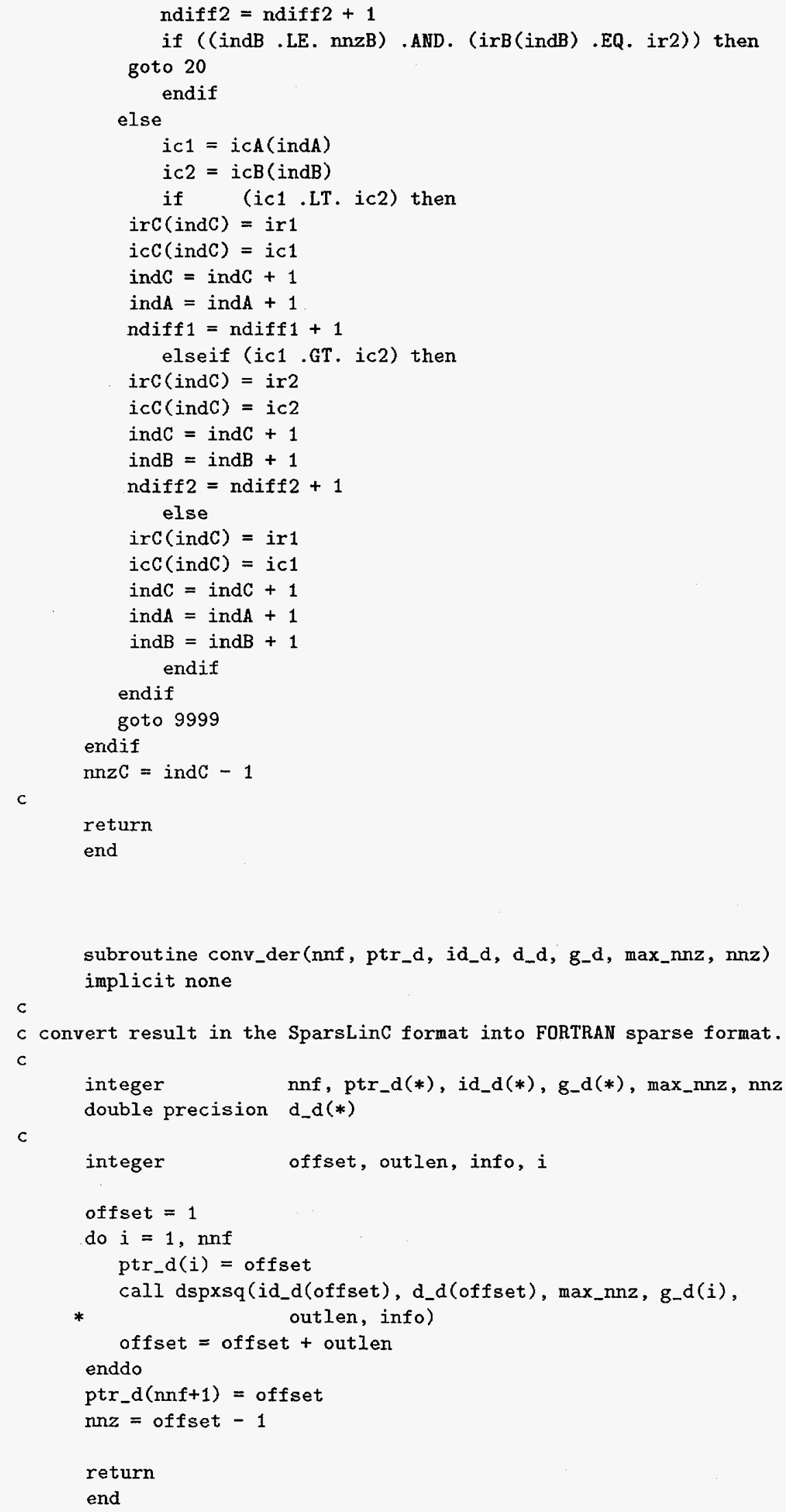




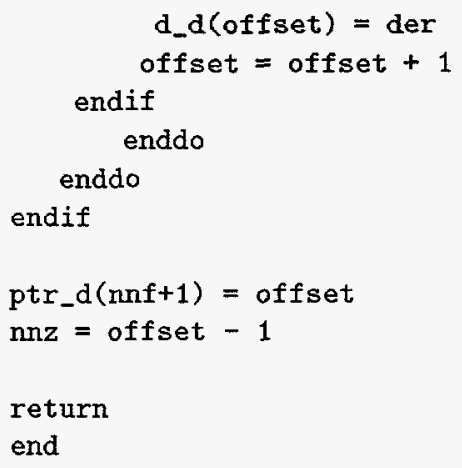

\section{G ADIC FormJacobian}

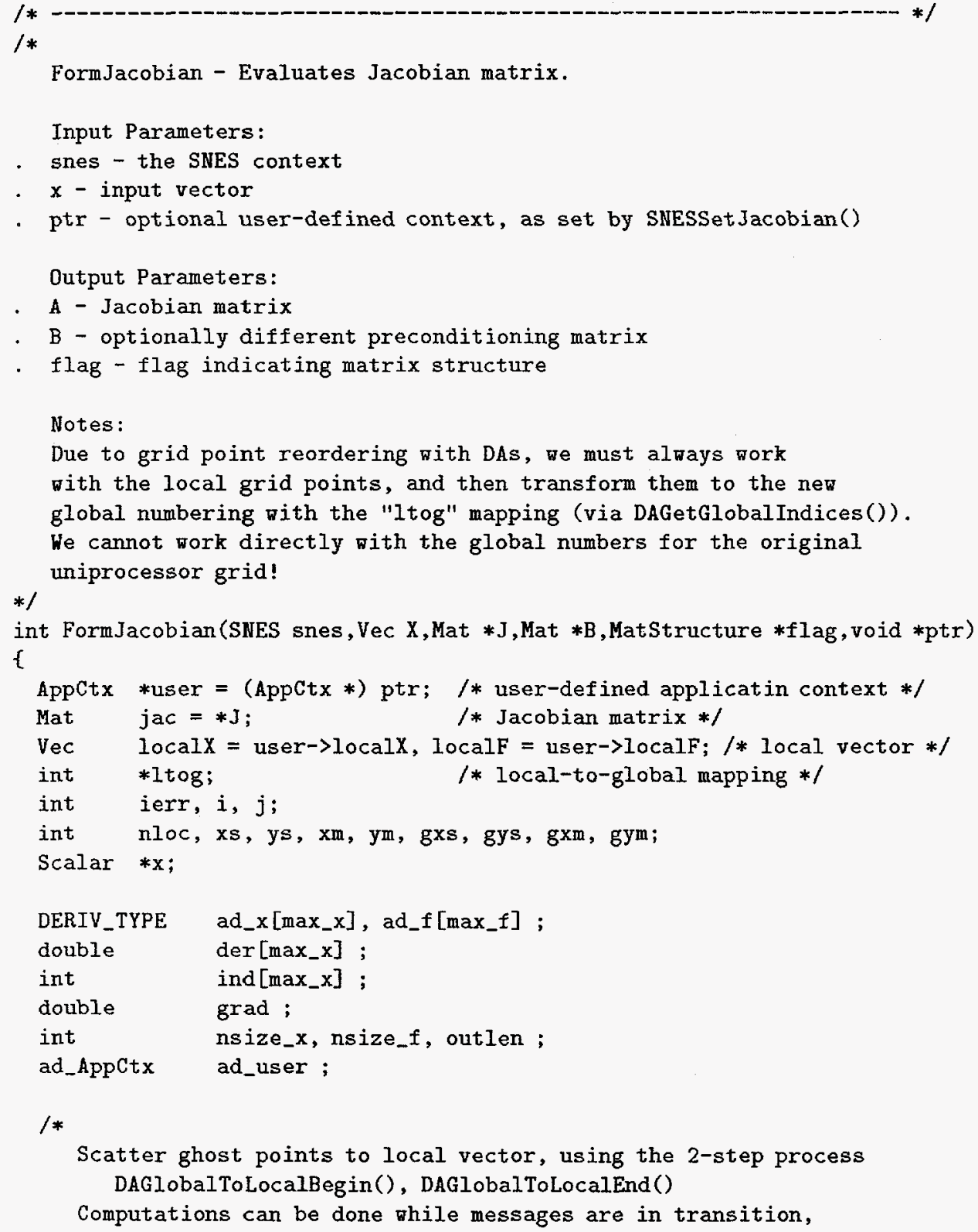




$$
\text { */ }
$$

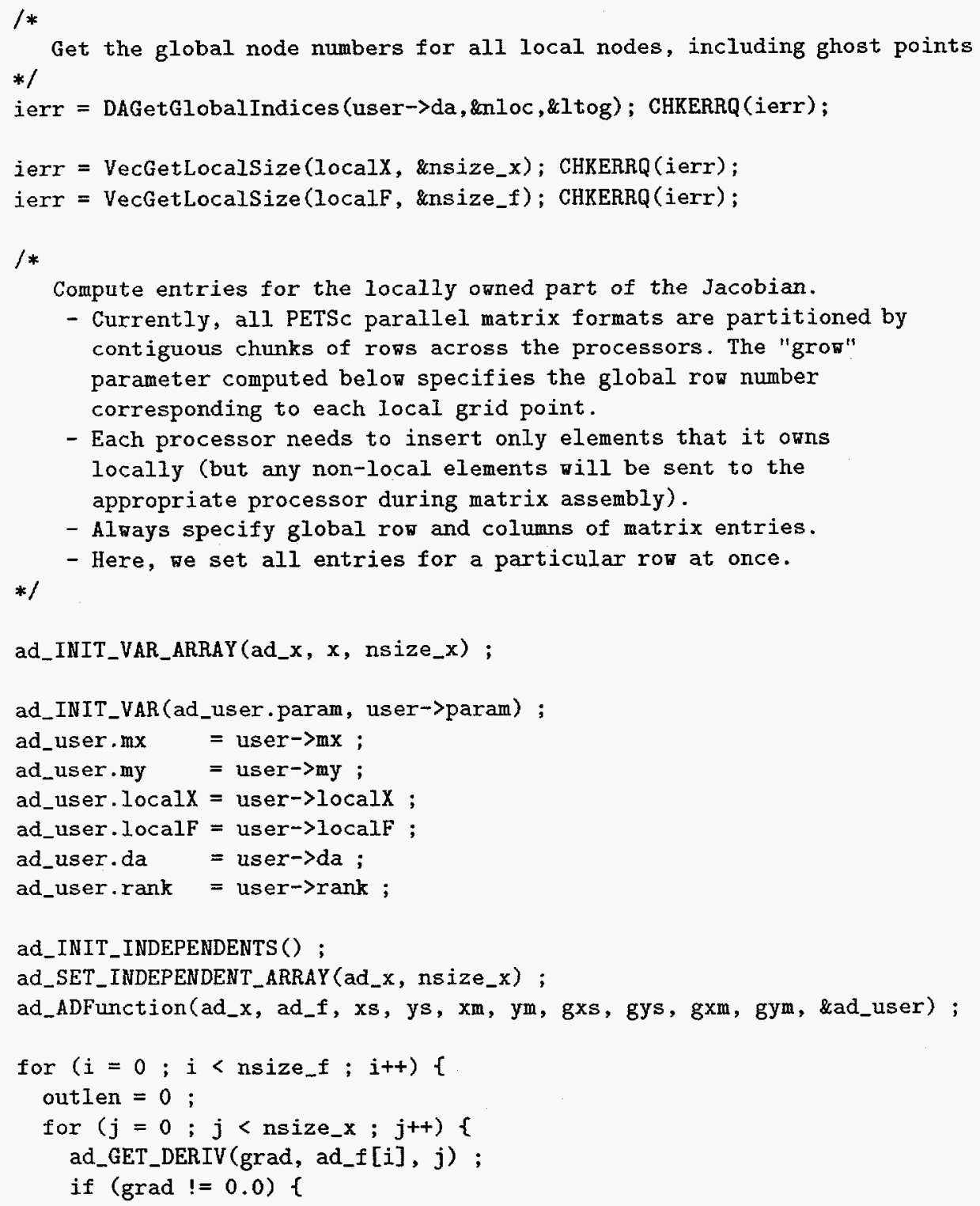




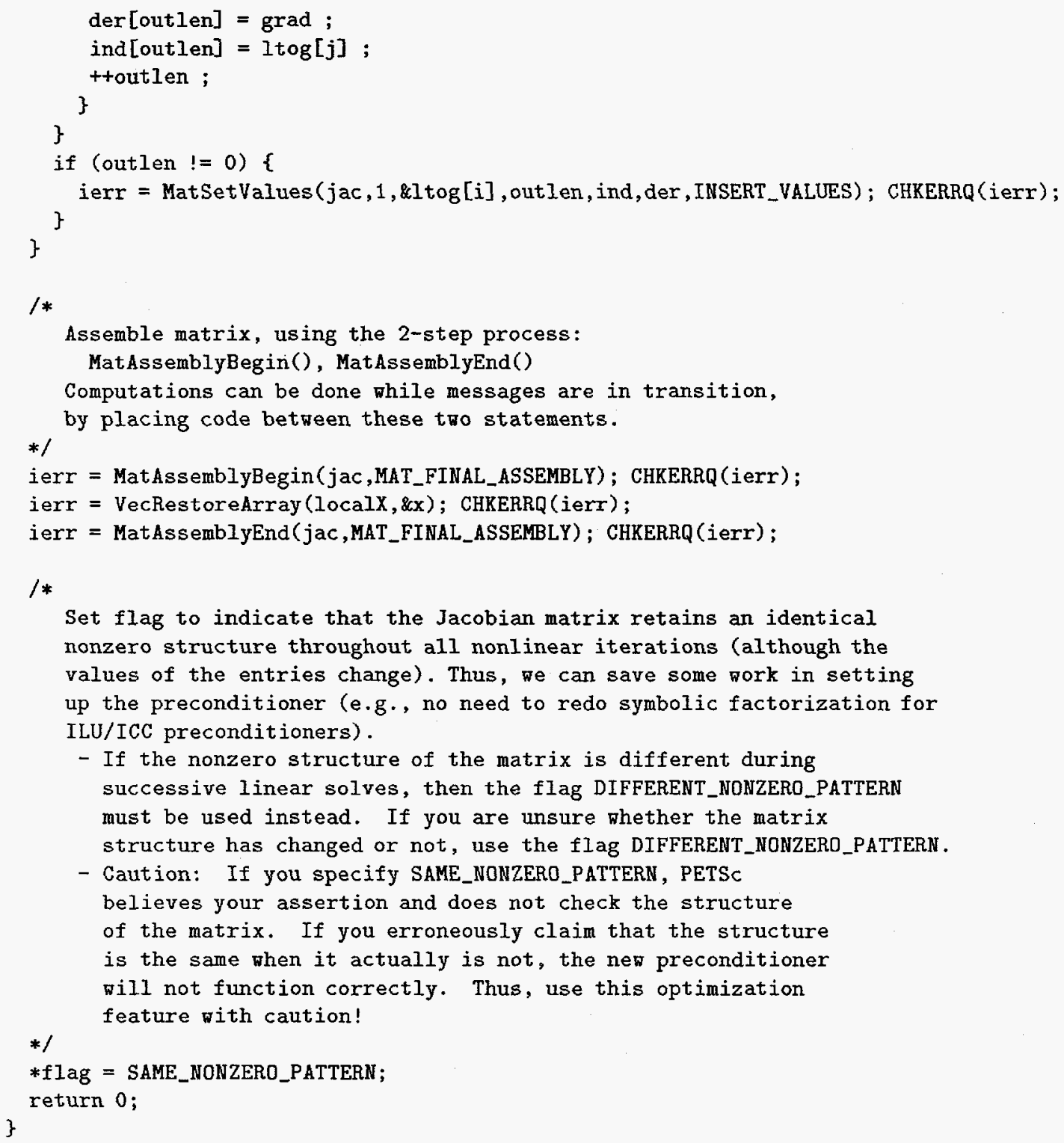




\section{References}

[1] B. Averick, J. Moré, C. Bischof, A. Carle, and A. Griewank. Computing large sparse Jacobian matrices using automatic differentiation. SIAM Journal on Scientific Computing, 15(2):285-294, 1994.

[2] S. Balay, W. D. Gropp, L. C. McInnes, and B. F. Smith. Efficient management of parallelism in objectoriented numerical software libraries. In E. Arge, A. M. Bruaset, and H. P. Langtangen, editors, Modern Software Tools for Scientific Computing. Birkhauser Boston, Cambridge, MA, 1997.

[3] C. Bischof, A. Carle, P. Khademi, and A. Mauer. ADIFOR 2.0: Automatic differentiation of Fortran 77 programs. IEEE Computational Science \& Engineering, 3(3):18-32, 1996.

[4] C. Bischof, A. Carle, P. Khademi, A. Mauer, and P. Hovland. ADIFOR 2.0 user's guide (Revision C). Technical Memorandum ANL/MCS-TM-192, Mathematics and Computer Science Division, Argonne National Laboratory, 1995.

[5] C. Bischof and L. Roh. ADIC user guide, 1996. Unpublished information, Argonne National Laboratory.

[6] C. Bischof, L. Roh, and A. Mauer. ADIC - An extensible automatic differentiation tool for ANSIC. Preprint ANL/MCS-P626-1196, Mathematics and Computer Science Division, Argonne National Laboratory, 1996. 